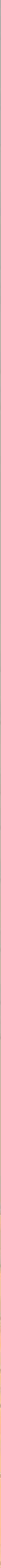




\title{
Del Pezzo surfaces and representation theory
}

\author{
Vera V. Serganova and Alexei N. Skorobogatov
}

To Yuri Ivanovich Manin on his seventieth birthday

\begin{abstract}
The connection between del Pezzo surfaces and root systems goes back to Coxeter and $\mathrm{Du}$ Val, and was given modern treatment by Manin in his seminal book Cubic forms. Batyrev conjectured that a universal torsor on a del Pezzo surface can be embedded into a certain projective homogeneous space of the semisimple group with the same root system, equivariantly with respect to the maximal torus action. Computational proofs of this conjecture based on the structure of the Cox ring have been given recently by Popov and Derenthal. We give a new proof of Batyrev's conjecture using an inductive process, interpreting the blowing-up of a point on a del Pezzo surface in terms of representations of Lie algebras corresponding to Hermitian symmetric pairs.
\end{abstract}

\section{Introduction}

Del Pezzo surfaces, classically defined as smooth surfaces of degree $d$ in the projective space $\mathbb{P}^{d}, d \geq 3$, are among the most studied and best understood algebraic varieties. Over an algebraically closed ground field such a surface is the quadric $\mathbb{P}^{1} \times \mathbb{P}^{1}$ or the projective plane $\mathbb{P}^{2}$ with $r=9-d$ points in general positions blown up. In this definition, $d$ can be any integer between 1 and 9. Despite the apparent simplicity the enumerative geometry of these surfaces displays amazing symmetries and puzzling coincidences. The 27 lines on a smooth cubic surface were discovered by Cayley and Salmon, and the symmetries of their configurations were explored by Schoutte, Coxeter and Du Val. Manin [1986] gave a modern exposition of this subject, with many geometric and arithmetic applications. He showed that the Picard group of a del Pezzo surface $X$ of degree $d=9-r$, where $d \leq 6$, contains a root system $\mathrm{R}_{r}$ of rank $r$ in such a way that the automorphism group of the incidence graph of the exceptional curves on $X$ is the Weyl group $\mathrm{W}\left(\mathrm{R}_{r}\right)$. These root systems are embedded into one another: $\mathrm{R}_{8}=\mathrm{E}_{8}$, and as $r$ decreases one chops one by one the nodes off the long end of the Dynkin diagram of $\mathrm{E}_{8}$, until the diagram

MSC2000: primary 14J26; secondary 17B25, 17B10.

Keywords: del Pezzo surface, homogeneous space, Lie algebra. 
becomes disconnected. Let $\alpha_{r}$ be the simple root of $\mathrm{R}_{r}$ corresponding to the node which must be removed from the Dynkin diagram of $\mathrm{R}_{r}$ in order to obtain that of $\mathrm{R}_{r-1}$; let $\omega_{r}$ be the fundamental weight dual to $\alpha_{r}$. For $r=4,5,6,7$ the number of exceptional curves on $X$ is $\left|\mathrm{W}\left(\mathrm{R}_{r}\right) / \mathrm{W}\left(\mathrm{R}_{r-1}\right)\right|=10,16,27,56$, respectively, and this is also the dimension of the irreducible minuscule representation $V\left(\omega_{r}\right)$ of the Lie algebra $\mathfrak{g}_{r}$ of type $\mathrm{R}_{r}$ with the highest weight $\omega_{r}$. It is tempting to try to recover the Lie algebra directly from a del Pezzo surface, but one has to bear in mind that the del Pezzo surfaces of degree $d \leq 5$ depend on $10-2 d$ moduli, so the Lie algebra should somehow take into account all del Pezzo surfaces of given degree; see [Manivel 2006], and also [Friedman and Morgan 2002; Leung 2000].

Universal torsors were introduced by Colliot-Thélène and Sansuc in the 1970's in a seemingly unrelated line of research; see [Colliot-Thélène and Sansuc 1987] or [Skorobogatov 2001]. If $X$ is a smooth projective variety over a field $k$, then an $X$-torsor under a torus $T$ is a pair $(Y, f)$, where $Y$ is a variety over $k$ with a free action of $T$, and $f$ is an affine morphism $Y \rightarrow X$ whose fibres are the orbits of $T$. An $X$-torsor is universal if all invertible regular functions on $Y$ are constant, and the Picard group of $Y$ is trivial (see Section 1 for details). Then $T$ is isomorphic to the Néron-Severi torus of $X$, that is, the algebraic torus dual to the Picard lattice of $X$ over an algebraic closure of $k$. In the work of Colliot-Thélène, Sansuc, Swinnerton-Dyer, Salberger and the second named author (see the references in [Skorobogatov 2001]) the birational geometry of universal torsors on del Pezzo surfaces of degrees 3 and 4 played a crucial role in gaining some understanding of the rational points on these surfaces over number fields, for example, the Hasse principle, weak approximation, the Brauer-Manin obstruction, and R-equivalence. The work of Batyrev, Tschinkel, Peyre, Salberger, Hassett, de la Bretèche, HeathBrown, Browning and others on the Manin-Batyrev conjecture on the number of rational points of bounded height, highlighted the importance of explicitly describing universal torsors as algebraic varieties, and not merely their birational structure. However, in the most interesting cases such as those of (smooth) del Pezzo surfaces of degrees 3 and 4, the explicit equations of universal torsors turned out to be quite complicated to write down.

Around 1990, Victor Batyrev told one of us (Skorobogatov) about his conjecture relating universal torsors on del Pezzo surfaces to certain projective homogeneous spaces. Let $G_{r}$ be a simply connected semisimple group of type $\mathrm{R}_{r}$. We fix a maximal torus $H_{r} \subset G_{r}$, and a basis of simple roots in the character group of $H_{r}$. Let $P_{r} \subset G_{r}$ be the maximal parabolic subgroup defined by the root $\alpha_{r}$ (the stabilizer of the line spanned by the highest weight vector of $\left.V\left(\omega_{r}\right)\right)$. Batyrev conjectured that a universal torsor $\mathcal{T}$ on a del Pezzo surface $X$ of degree $d=9-r$ over an algebraically closed field can be embedded into the affine cone $\left(G_{r} / P_{r}\right)_{a} \subset V\left(\omega_{r}\right)$ over $G_{r} / P_{r}$, equivariantly with respect to the action of the Néron-Severi torus $T_{r}$ 
of $X$, identified with an extension of $H_{r}$ by the scalar matrices $\mathbf{G}_{m}$. Moreover, the exceptional curves on $X$ should be the images of the weight hyperplane sections of $\mathscr{T}$, that is, the intersections of $\mathscr{T}$ with the $H_{r}$-invariant hyperplanes in $V\left(\omega_{r}\right)$. Inspired by these ideas, one of us showed in [Skorobogatov 1993] that the set of stable points of the affine cone over the Grassmannian $G(3,5)$ with respect to the action of the diagonal torus of SL(5), is a universal torsor over the del Pezzo surface of degree 5 which is the geometric invariant theory (GIT) quotient by this action. Batyrev's line of attack on the general case of his conjecture uses the Cox ring of $X$, which can be interpreted as the ring of regular functions on a universal torsor over X. Indeed, Batyrev and Popov [2004] (see also [Derenthal 2006]) found the generators and the relations of the Cox ring, which enabled Popov in his thesis [2001] in the case $d=4$ and Derenthal [2007] in the cases $d=3$ and $d=2$ to prove Batyrev's conjecture by identifying the generators with the weights of $V\left(\omega_{r}\right)$, and comparing the relations with the well-known equations of $G_{r} / P_{r}$. The proofs of [Popov 2001] and [Derenthal 2007] are based on a substantial amount of calculation which grows rapidly with $r$, and do not seem to give much insight into why things work this way.

Here we prove Batyrev's conjecture for del Pezzo surfaces of degrees 2 to 4 using a totally different approach, the representation theory of Lie algebras. We start with the known case of a del Pezzo surface of degree 5. (Alternatively, one could start with the simpler though somewhat irregular case of degree 6; see [Batyrev and Popov 2004].) Let $\mathfrak{p}_{r}$ be the Lie algebra of $P_{r} \subset G_{r}$. We build an inductive process based on the fact that the pair $\left(\mathrm{R}_{r}, \alpha_{r}\right)$ for $r=4,5,6,7$ is a Hermitian symmetric pair, that is, the complementary nilpotent algebra of $\mathfrak{p}_{r}$ in $\mathfrak{g}_{r}$ is commutative. We show that $V\left(\omega_{r}\right)$, as a $\mathfrak{g}_{r-1}$-module, has a direct factor isomorphic to $V\left(\omega_{r-1}\right)$, and that the restriction of the projection $V\left(\omega_{r}\right) \rightarrow V\left(\omega_{r-1}\right)$ to a certain open subset $U \subset\left(G_{r} / P_{r}\right)_{a}$ is the composition of a $\mathbf{G}_{m}$-torsor and a morphism inverse to the blowing-up of $V\left(\omega_{r-1}\right) \backslash\{0\}$ at $\left(G_{r-1} / P_{r-1}\right)_{a} \backslash\{0\}$ (see Corollary 4.2). Now we can explain the main idea of our proof. Suppose that a universal torsor $\mathscr{T}$ over a del Pezzo surface $X$ of degree $9-(r-1)$ is $T_{r-1}$-equivariantly embedded into the affine cone $\left(G_{r-1} / P_{r-1}\right)_{a} \subset V\left(\omega_{r-1}\right)$. Let $M$ be a point on $X$ outside of the exceptional curves, and $\mathrm{Bl}_{M}(X)$ the blowing-up of $X$ at $M$. The space $V\left(\omega_{r-1}\right)$ is a direct sum of 1-dimensional weight spaces of $H_{r-1}$, so the torus consisting of the diagonal matrices with respect to a weight basis of $V\left(\omega_{r-1}\right)$ does not depend on the choice of this basis. We show how to choose an element $t_{M}$ of this torus so that the translation $t_{M}^{-1}\left(G_{r-1} / P_{r-1}\right)_{a}$ intersects $\mathcal{T}$ exactly in the fibre of $f: \mathcal{T} \rightarrow X$ over $M$. Then the closure of the inverse image of $t_{M}\left(\mathscr{T} \backslash f^{-1}(M)\right)$ in $U$ is a universal torsor over $\mathrm{Bl}_{M}(X)$. This yields a $T_{r}$-equivariant embedding of this universal torsor into $\left(G_{r} / P_{r}\right)_{a}$. We then show that the image of this embedding is contained in the 


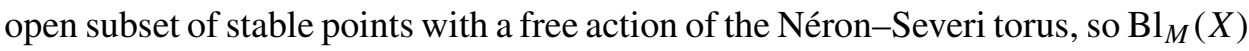
embeds into the corresponding quotient.

Here is the structure of the paper. In Section 1 we recall equivalent definitions and some basic properties of universal torsors. In Section 2 we prove that the left action of a maximal torus of $G$ on $G / P$, where $P$ is a maximal parabolic subgroup of a semisimple algebraic group $G$, turns the set of stable points with free action of the maximal torus into a universal torsor on an open subset of the GIT quotient of $G / P$ by this action (with an explicit list of exceptions, see Theorem 2.7 for the precise statement). In Section 3 we recall the necessary background from the representation theory of Lie algebras. The implications for the structure of the projection of $\left(G_{r} / P_{r}\right)_{a}$ to $V\left(\omega_{r-1}\right)$ are studied in Section 4. In Section 5 we list some well-known properties of del Pezzo surfaces. Our main result, Theorem 6.1, is stated and proved in Section 6.

\section{Universal torsors}

Let $k$ be a field of characteristic 0 with an algebraic closure $\bar{k}$. Let $X$ be a geometrically integral variety over $k$. We write $\bar{X}$ for $X \times_{k} \bar{k}$. We denote by $\bar{k}[X]$ the $\bar{k}$-algebra of regular functions on $\bar{X}$, and by $\bar{k}[X]^{*}$ the group of its invertible elements.

Let $T$ be an algebraic $k$-torus, that is, an algebraic group such that $\bar{T} \simeq \mathbf{G}_{m}^{n}$ for some $n$. Let $\hat{T} \simeq \mathbf{Z}^{n}$ be the group of characters of $T$. The Galois group $\Gamma=\operatorname{Gal}(\bar{k} / k)$ naturally acts on $\hat{T}$.

For generalities on torsors the reader is referred to [Skorobogatov 2001]. An $X$-torsor under $T$ is a pair $(\mathcal{T}, f)$, where $\mathscr{T}$ is a $k$-variety with an action of $T$, and $f: \mathscr{T} \rightarrow X$ is a morphism such that locally in étale topology $\mathscr{T} \rightarrow X$ is $T$ equivariantly isomorphic to $X \times_{k} T$. The following lemma is well known.

Lemma 1.1. Suppose that a $k$-torus $T$ acts on a $k$-variety $Y$ with trivial stabilizers, and $g: Y \rightarrow X$ is an affine morphism of $k$-varieties, whose fibres are orbits of $T$. Then $g: Y \rightarrow X$ is a torsor under $T$.

Proof. The property of $g$ to be a torsor can be checked locally on $X$. Let $U$ be an open affine subset of $X$. Since $g$ is affine, $g^{-1}(U)$ is also affine [Hartshorne 1977, II, 5, Exercise 5.17]. Since the stabilizers of all $\bar{k}$-points of $g^{-1}(U)$ are trivial, by a corollary of Luna's étale slice theorem [Mumford et al. 1994, p. 153] the natural map $g^{-1}(U) \rightarrow U$ is a torsor under $T$. The lemma follows.

Colliot-Thélène and Sansuc associated to a torsor $f: \mathcal{T} \rightarrow X$ under a torus $T$ the exact sequence of $\Gamma$-modules [Colliot-Thélène and Sansuc 1987, 2.1.1]

$$
1 \rightarrow \bar{k}[X]^{*} / \bar{k}^{*} \rightarrow \bar{k}[\mathscr{T}]^{*} / \bar{k}^{*} \rightarrow \hat{T} \rightarrow \operatorname{Pic} \bar{X} \rightarrow \operatorname{Pic} \overline{\mathscr{T}} \rightarrow 0 .
$$


Here the second and fifth arrows are induced by $f$. The fourth arrow is called the type of $\mathscr{T} \rightarrow X$. To define it, consider the natural pairing compatible with the action of the Galois group $\Gamma$,

$$
\cup: \mathrm{H}^{1}(\bar{X}, T) \times \hat{T} \rightarrow \mathrm{H}^{1}\left(\bar{X}, \mathbf{G}_{m}\right)=\operatorname{Pic} \bar{X},
$$

where the cohomology groups are in étale or Zariski topology. The type sends $\chi \in \hat{T}$ to $[\overline{\mathscr{T}}] \cup \chi$, where $[\overline{\mathscr{T}}] \in \mathrm{H}^{1}(\bar{X}, T)$ is the class of the torsor $\overline{\mathscr{T}} \rightarrow \bar{X}$. A torsor $\mathcal{T} \rightarrow X$ is called universal if its type is an isomorphism. If $X$ is projective, Equation (1) gives the following characterization of the universal torsors: an $X$ torsor under a torus is universal if and only if Pic $\overline{\mathscr{T}}=0$ and $\bar{k}[\mathscr{T}]^{*}=\bar{k}^{*}$, that is, $\overline{\mathscr{T}}$ has no nonconstant invertible regular functions.

We now give an equivalent definition of type which does not involve cohomology. Let $K=\bar{k}(X)$ be the function field of $\bar{X}$, and $\mathscr{T}_{K}$ the generic fibre of $\overline{\mathscr{T}} \rightarrow \bar{X}$. By Hilbert's Theorem 90, the $K$-torsor $\mathscr{T}_{K}$ is trivial, that is, is isomorphic to $T_{K}=T \times{ }_{k} K$. By Rosenlicht's lemma we have an isomorphism of $\Gamma$-modules

$$
K\left[\mathscr{T}_{K}\right]^{*} / K^{*}=K\left[T_{K}\right]^{*} / K^{*}=\hat{T} .
$$

This isomorphism associates to a character $\chi \in \hat{T}$ a rational function $\phi \in \bar{k}(\mathscr{T})^{*}$ such that $\phi(t x)=\chi(t) \phi(x)$; the function $\phi$ is well defined up to an element of $K^{*}=\bar{k}(X)^{*}$. The divisor of $\phi$ on $\overline{\mathscr{T}}$ does not meet the generic fibre $\mathscr{T}_{K}$, and hence comes from a divisor on $\bar{X}$ defined up to a principal divisor. We obtain a well-defined class $\tau(\chi)$ in Pic $\bar{X}$.

Lemma 1.2. The map $\tau: \hat{T} \rightarrow \operatorname{Pic} \bar{X}$ coincides with the type of $f: \mathscr{T} \rightarrow X$ up to sign.

Proof. According to [Skorobogatov 2001, Lemma 2.3.1 (ii)], the type associates to $\chi$ the subsheaf $O_{\chi}$ of $\chi$-semiinvariants of the sheaf $f_{*}\left(\mathscr{O}_{\mathscr{T}}\right)$. The function $\phi$ is a rational section of $\mathrm{O}_{\chi}$; hence, the class of its divisor represents $\mathrm{O}_{\chi} \in \operatorname{Pic} \bar{X}$.

For the sake of completeness we note that if $f: \mathcal{T} \rightarrow X$ is a universal torsor, then the group of divisors on $\bar{X}$ is naturally identified with $K\left[\mathscr{T}_{K}\right]^{*} / \bar{k}^{*}$; this identifies the semigroup of effective divisors on $\bar{X}$ with $\left(K\left[\mathscr{T}_{K}\right]^{*} \cap \bar{k}[\mathcal{T}]\right) / \bar{k}^{*}$.

We have

$$
\bar{k}[\mathscr{T}]=\bigoplus_{\chi \in \hat{T}} \bar{k}[\mathscr{T}]_{\chi},
$$

where $\bar{k}[\mathscr{T}]_{\chi}$ is the set of regular functions $\phi$ on $\mathscr{T}$, satisfying $\phi(t x)=\chi(t) \phi(x)$ for any $t$ in $T$. We also define $\bar{k}(\mathscr{T})_{\chi}$ as the set of rational functions on $\mathscr{T}$, satisfying the same condition. Since $\bar{k}(\mathscr{T})_{\chi}$ is the group of rational sections of the sheaf $O_{\chi}$, we have $\bar{k}[\mathscr{T}]_{\chi}=\mathrm{H}^{0}\left(\bar{X}, O_{\chi}\right)$. Hence if the sheaf $O_{\chi}$ defines a morphism

$$
X \rightarrow \mathbb{P}\left(\mathrm{H}^{0}\left(\bar{X}, \mathrm{O}_{\chi}\right)^{*}\right),
$$


we obtain a commutative diagram

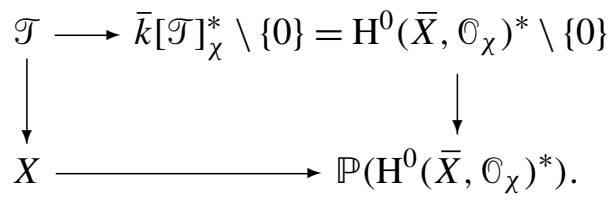

Here the asterisk denotes the dual vector space.

\section{2. $G / P$ and the torus quotient}

Let $G$ be a split simple simply connected algebraic group over $k$, with a split maximal torus $H \subset G$; in this case the root system $\mathrm{R}$ of $G$ relative to $H$ is irreducible. Write $\hat{H}$ for the character group of $H$. We use the standard notation $Q(\mathrm{R})$ for the lattice generated by the simple roots, then $P(\mathrm{R})=\hat{H}$ is the dual lattice generated by the fundamental weights. We denote the Weyl group by $\mathrm{W}=\mathrm{W}(\mathrm{R})$.

Let $G \rightarrow \mathrm{GL}(V)$ be an irreducible representation of $G$ with a fundamental highest weight $\omega \in \hat{H}$. Let $v \in V$ be a highest weight vector. The stabilizer of the line $k v$ is a maximal parabolic subgroup $P \subset G$. The homogeneous space $G / P$ is thus a smooth projective subvariety of $\mathbb{P}(V)$, which is indeed the only closed orbit of $G$ in $\mathbb{P}(V)$. We write $\hat{P}$ (respectively, $\hat{G}$ ) for the character group of $P$ (respectively, of $G)$. Let $\varepsilon: \hat{P} \rightarrow$ Pic $G / P$ be the map associating to the character $\chi \in \hat{P}$ the $G / P$-torsor under $\mathbf{G}_{m}$ defined as the quotient of $G \times \mathbf{G}_{m}$ by $P$, where $p \in P$ sends $(g, t)$ to $\left(g p^{-1}, \chi(p) t\right)$. This map fits into the exact sequence

$$
0 \rightarrow \hat{G} \rightarrow \hat{P} \rightarrow \operatorname{Pic} G / P \rightarrow \operatorname{Pic} G \rightarrow 0 .
$$

Since $G$ is semisimple and simply connected we have $\hat{G}=\operatorname{Pic} G=0$ so $\varepsilon$ is an isomorphism; see, for example, [Popov 1974]. Since $\hat{P}$ is the subgroup of $\hat{H}$ generated by $\omega$, we see that $\operatorname{Pic} G / P$ is generated by the hyperplane section class. This fact implies the following elementary statement from projective geometry.

Lemma 2.1. Let $L_{1}$ and $L_{2}$ be distinct hyperplanes in the projective space $\mathbb{P}(V)$. Then $(G / P) \cap L_{1} \cap L_{2}$ has codimension 2 in $G / P$.

Proof. Since Pic $G / P$ is generated by the class of a hyperplane section, for any hyperplane $L \subset \mathbb{P}(V)$ the closed subset $(G / P) \cap L$ is irreducible of codimension 1 , and the intersection has multiplicity 1 . If the codimension of $(G / P) \cap L_{1} \cap L_{2}$ in $G / P$ is 1 , we have $(G / P) \cap L_{1} \cap L_{2}=(G / P) \cap L$ for any $L$ in the linear family spanned by $L_{1}$ and $L_{2}$. Choosing $L$ passing through a point of $G / P$ not contained in $L_{1}$, we deduce a contradiction.

By the irreducibility of $V$ the center $Z(G)$ acts diagonally on $V$, and hence it acts trivially on $\mathbb{P}(V)$. For a $\bar{k}$-point $x \in \mathbb{P}(V)$ we denote the stabilizer of $x$ in $H$ by $\operatorname{St}_{H}(x)$. We now show that for $x$ in a dense open subset of $G / P$ we have 
$\mathrm{St}_{H}(x)=Z(G)$, and determine the points such that $\mathrm{St}_{H}(x)$ is strictly bigger than $Z(G)$.

Proposition 2.2. Let $x$ be a $\bar{k}$-point of $G / P$, and let $K_{x}$ be the connected component of the centralizer of $\mathrm{St}_{H}(x)$ in $G$. Then we have the following properties.

(i) $K_{x}$ is a reductive subgroup of $G, H \subset K_{x}$;

(ii) $x \in K_{x} w v=K_{x} /\left(w P w^{-1} \cap K_{x}\right)$ for some $w \in \mathrm{W}$;

(iii) $Z\left(K_{x}\right)=\mathrm{St}_{H}(x)$;

(iv) $\mathrm{St}_{H}(x)$ is finite if and only if $K_{x}$ is semisimple, in which case the ranks of $K_{x}$ and $G$ are equal.

Proof. If $\mathrm{St}_{H}(x)=Z(G)$, then $K_{x}=G$, and all the statements are clearly true. Assume that $\operatorname{St}_{H}(x)$ is bigger than $Z(G)$, then $K_{x}$ is a closed subgroup of $G$, $K_{x} \neq G$.

Let $\mathfrak{k}_{x}$ be the Lie algebra of $K_{x}$; explicitly $\mathfrak{k}_{x} \subset \mathfrak{g}$ is the fixed set of $\left.\operatorname{Ad}_{\left(\operatorname{St}_{H}\right.}(x)\right)$. Since $\mathfrak{k}_{x}$ contains the Cartan subalgebra $\mathfrak{h}$, it has a root decomposition

$$
\mathfrak{k}_{x}=\bigoplus_{\alpha \in S} \mathfrak{g}_{\alpha}
$$

where $S \subset \mathrm{R}$. Let $\exp _{\alpha} \in \hat{H}$ be the multiplicative character defined by the root $\alpha \in \mathrm{R}$. The space $\mathfrak{g}_{\alpha}$ consists of $y \in \mathfrak{g}$ such that $\operatorname{Ad}(h) y=\exp _{\alpha}(h) y$ for all $h \in H$. Thus $\mathfrak{g}_{\alpha} \subset \mathfrak{k}_{x}$ if and only if $\operatorname{St}_{H}(x) \subset H$ is in the kernel of $\exp _{\alpha}$. Therefore $S=-S$, so $\mathfrak{k}_{x}$ is reductive, and hence so is $K_{x}$.

The fixed points of $H$ in $G / P$ come from the points $w v$, where $w \in \mathrm{W}$. One of these, say $x_{0}=w v$, is contained in the closure of the orbit $H x$. The stabilizer of $x_{0}$ in $G$ is the parabolic subgroup $w P w^{-1}$. To prove (ii) we need to show that $x$ belongs to the $K_{x}$-orbit of $x_{0}$. Let $N \subset G$ be the unipotent subgroup complementary to $w P w^{-1}$, such that the corresponding Lie algebras satisfy $\mathfrak{g}=\mathfrak{n} \bigoplus w \mathfrak{p} w^{-1}$. Then $N \cap w P w^{-1}=\{1\}$, and the $N$-orbit of the line $k x_{0}$ is the open Schubert cell $N x_{0} \subset G / w P w^{-1} \simeq G / P$. The intersection of this open Schubert cell with $H x$ is a nonempty open subset of $H x$; thus there is a $\bar{k}$-point $x_{1} \in H x \cap N x_{0}$. We can write $x_{1}=u . x_{0}$ for some $u \in N$. The complement to the union of connected components of the centralizer of $\mathrm{St}_{H}(x)$ other than $K_{x}$, is an open neighborhood of 1 in $G$. We choose $x_{1}$ in such a way that $u$ belongs to this open set. Since $H \subset K_{x}$, the points $x$ and $x_{1}$ are in the same $K_{x}$-orbit, so it is enough to show that $x_{1} \in K_{x} x_{0}$. Any $t \in \mathrm{St}_{H}(x)$ fixes both $x_{1}$ and $x_{0}$; thus $x_{1}=u \cdot x_{0}=t^{-1} u t . x_{0}$. Therefore, $u^{-1} t^{-1} u t$ fixes $x_{0}$; hence $u^{-1} t^{-1} u t \in w P w^{-1}$. On the other hand, $H$ normalizes $N$; thus $t^{-1} u t \in N$, implying $u^{-1} t^{-1} u t \in N$. Since the intersection of $w P w^{-1}$ and $N$ is $\{1\}$, we see that $u$ and $t$ commute. By the choice of $x_{1}$ we 
see that $u$ is in the connected component of 1 of the centralizer of $\operatorname{St}_{H}(x)$, that is, $u \in K_{x}$. This completes the proof of (ii).

The center of $K_{x}$ is contained in every maximal torus, in particular, in $H$. Any element of $Z\left(K_{x}\right)$ fixes $x$, since $x \in K_{x} /\left(w P w^{-1} \cap K_{x}\right)$, so $Z\left(K_{x}\right) \subset \operatorname{St}_{H}(x)$. On the other hand, every element of $\mathrm{St}_{H}(x)$ commutes with $K_{x}$ by the definition of $K_{x}$. But $\mathrm{St}_{H}(x) \subset H \subset K_{x}$; hence $\mathrm{St}_{H}(x) \subset Z\left(K_{x}\right)$. This proves (iii).

The rank of the semisimple part of $K_{x}$ equals the rank of $G$ if and only if $Z\left(K_{x}\right)$ is finite. If $Z\left(K_{x}\right)$ is finite, then $K_{x}$ is semisimple by definition. Thus (iv) follows from (iii).

Fix a weight basis in $V$, that is, a basis in which $H$ is diagonal. The weight of a coordinate is the character of $H$ by which $H$ acts on it. Denote by $\Lambda$ the set of weights of $H$ in $V$, and by $\operatorname{wt}(x)$ the set of weights of $x \in G / P$, that is, the weights of the nonvanishing coordinates of $x$.

Corollary 2.3. Assume that $\mathrm{R}$ is simply laced. Then the codimension of the set of $\bar{k}$-points $x \in G / P$ such that $\mathrm{St}_{H}(x)$ is finite, and $\mathrm{St}_{H}(x) \neq Z(G)$, is at least 2.

Proof. By Proposition 2.2 and $\mathrm{W}$-invariance it is sufficient to show that the codimension of $K v$ in $G v$ is at least 2 for any proper connected semisimple subgroup $K \subset G$ containing $H$. The set of such subgroups is clearly finite.

For any $x \in G / P$ the property $\mathrm{wt}(x)=\Lambda$ implies $\operatorname{St}_{H}(x)=Z(G)$. Let $V^{\prime} \subset V$ be the irreducible representation of $K$ generated by $v$. Denote by $\Lambda^{\prime}$ the set of weights of $V^{\prime}$, and write $V=V^{\prime} \oplus U$, where $U$ is another $K$-invariant subspace. First, we claim that $\Lambda^{\prime} \neq \Lambda$ because otherwise one can find $x \in \mathbb{P}(K v)$ such that $\operatorname{wt}(x)=\Lambda$, and $\operatorname{St}_{H}(x)=Z(G)=Z(K)$ would imply $K=G$. In particular, $U \neq 0$. If $\operatorname{dim} U>1$, then the codimension of $K v \subset G v \cap V^{\prime}$ is at least 2 by Lemma 2.1.

If $\operatorname{dim} U=1$, then $U$ is a trivial representation of $K$ and 0 is not a weight of $V^{\prime}$. But then $U$ is invariant under the action of the Weyl group $\mathrm{W}$. Therefore $w K w^{-1}$ acts trivially on $U$ for any $w \in \mathrm{W}$. If $a \in \mathrm{R}$ is a root of $K$, then $w(a)$ is a root of $w K w^{-1}$. But in the simply laced case $\mathrm{W}$ acts transitively on $\mathrm{R}$; hence, the subgroups $w K w^{-1}, w \in \mathrm{W}$, generate the whole group $G$. Thus, $U$ is $G$-invariant, but that contradicts the irreducibility of $V$.

Recall that a $\bar{k}$-point $x \in V$ is called stable for the action of $H$ if the orbit $H x$ is closed, and the stabilizer of $x$ in $H$ is finite [Mumford et al. 1994, p. 194]. We always consider the stability with respect to the action of $H$, and drop the reference to $H$ when it causes no confusion.

For a subset $M \subset \hat{H}$ we write $\operatorname{Conv}(M)$ for the convex hull of $M$ in the vector space $\hat{H} \otimes \mathbb{R}$. It is well known that $\operatorname{Conv}(\Lambda)=\operatorname{Conv}(\mathrm{W} \omega)$ [Gel'fand and Serganova 1987; Flaschka and Haine 1991]; see [Dabrowski 1996, Proposition 2.2 (i)] for a short proof. The Hilbert-Mumford numerical criterion of stability says that $x$ is 
stable if and only if 0 belongs to the interior of $\operatorname{Conv}(w t(x))$ [Dolgachev 2003, Theorem 9.2].

In the following statement and thereafter the numeration of the nodes of Dynkin diagrams, simple roots and fundamental weights follows the conventions of [Bourbaki 1981].

Proposition 2.4. Assume that the pair $(\mathrm{R}, \omega)$ is not in the following list:

$$
\left(\mathrm{R}_{r}, \omega_{1}\right),\left(\mathrm{A}_{r}, \omega_{r}\right),\left(\mathrm{A}_{3}, \omega_{2}\right),\left(\mathrm{B}_{2}, \omega_{2}\right),\left(\mathrm{C}_{2}, \omega_{2}\right),\left(\mathrm{D}_{4}, \omega_{3}\right),\left(\mathrm{D}_{4}, \omega_{4}\right),
$$

where $\mathrm{R}_{r}$ is $\mathrm{A}_{r}, \mathrm{~B}_{r}, \mathrm{C}_{r}$, or $\mathrm{D}_{r}$. Let $x$ be a point of $V \otimes_{k} \bar{k}$ such that no two elements of $\mathrm{W} \omega \backslash \mathrm{wt}(x)$ are adjacent vertices of $\operatorname{Conv}(\mathrm{W} \omega)$. Then $x$ is stable. In particular, the set of unstable points of $G / P$ has codimension at least 2.

Proof. Since $\sum_{w \in \mathrm{W}} w \omega=0$, the point 0 is contained in the interior of

$$
\operatorname{Conv}(\mathrm{W} \omega)=\operatorname{Conv}(\Lambda)
$$

in $\hat{H} \otimes \mathbb{R}$. Thus if all the coordinates of $x$ with weights in $\mathrm{W} \omega$ are nonzero, then $x$ is stable.

Now assume that exactly one such coordinate of $x$ is zero; because of the action of $\mathrm{W}$ it is no loss of generality to assume that it corresponds to $\omega$. The dimension of the corresponding eigenspace is 1 , so to check that $x$ is stable it is enough to show that 0 lies in the interior of $\operatorname{Conv}(\mathrm{W} \omega \backslash\{\omega\})$. The vertices of $\operatorname{Conv}(\mathrm{W} \omega)$ adjacent to $\omega$ are $\omega-w \alpha$, where $\alpha$ is the root dual to $\omega$, for all $w$ in the stabilizer of $\omega$ in W [Flaschka and Haine 1991, Lemma 3 and Cor. 2]. All these are contained in the hyperplane $L=0$, where

$$
L(y)=(y, \omega)-\left(\omega^{2}\right)+(\omega, \alpha)=(y, \omega)-\left(\omega^{2}\right)+\frac{1}{2}\left(\alpha^{2}\right) .
$$

We have $L(\omega)>0$. Thus 0 belongs to the interior of $\operatorname{Conv}(\mathrm{W} \omega \backslash\{\omega\})$ if and only if $\omega$ and 0 are separated by this hyperplane, that is, if and only if $L(0)<0$. Therefore, we need to check the condition

$$
\left(\omega^{2}\right)>\frac{1}{2}\left(\alpha^{2}\right) .
$$

Note that the numbers $2\left(\omega^{2}\right) /\left(\alpha^{2}\right)$, for all possible fundamental weights, are the diagonal elements of the inverse Cartan matrix of $\mathrm{R}$. A routine verification using the tables of [Bourbaki 1981] or [Onishchik and Vinberg 1990] shows that this inequality is satisfied for the pairs $(\mathrm{R}, \omega)$ not in the list (3).

Finally, let $\mathrm{W} \omega \backslash \operatorname{wt}(x)=\left\{\lambda_{1}, \ldots, \lambda_{n}\right\}$. By assumption $\lambda_{1}, \ldots, \lambda_{n}$ correspond to pairwise nonadjacent vertices of $\operatorname{Conv}(\mathrm{W} \omega)$. Thus

$$
\operatorname{Conv}\left(\mathrm{W} \omega \backslash\left\{\lambda_{1}, \ldots, \lambda_{n}\right\}\right)=\bigcap_{i=1}^{n} \operatorname{Conv}\left(\mathrm{W} \omega \backslash\left\{\lambda_{i}\right\}\right) .
$$


Since 0 is in the interior of each convex hull on the right hand side, it is also in the interior of $\operatorname{Conv}(\operatorname{wt}(x))$.

The last statement is an application of Lemma 2.1.

Definition 2.5. Let $T \subset \mathrm{GL}(V)$ be the torus generated by the image of $H$ in GL( $V)$ and the scalar matrices $\mathbf{G}_{m} \subset \mathrm{GL}(V)$. We write $(G / P)_{a}$ for the affine cone over $G / P$ in $V$, and $(G / P)_{a}^{s f}$ for the open subset of stable points with trivial stabilizers in $T$.

By the irreducibility of $V$, the stabilizer of $x \in V \otimes_{k} \bar{k}, v \neq 0$, in $T$ is trivial if and only if $\mathrm{St}_{H}(\operatorname{pr}(x))=Z(G)$, where $\operatorname{pr}(x)$ is the image of $x$ in $\mathbb{P}(V)$.

Lemma 2.6. There exist a smooth quasiprojective variety $Y$ and an affine morphism $f:(G / P)_{a}^{s f} \rightarrow Y$ which is a torsor with structure group $T$ with respect to its natural left action on $G / P$.

Proof. By geometric invariant theory there exist a quasiprojective variety $Y$ and an affine morphism $f:(G / P)_{a}^{s f} \rightarrow Y$ such that every fibre of $f$ is an orbit of $T$ [Mumford et al. 1994, Theorem 1.10 (iii)]. Since the stabilizers of all $\bar{k}$-points of $(G / P)_{a}^{s f}$ are trivial, Lemma 1.1 implies that $f:(G / P)_{a}^{s f} \rightarrow Y$ is a torsor under $T$. The smoothness of $Y$ follows from the smoothness of $(G / P)_{a}$, since a torsor is locally trivial in étale topology.

Theorem 2.7. Assume that the root system $\mathrm{R}$ is simply laced, and the pair $(\mathrm{R}, \omega)$ is not in the list (3). Then the only invertible regular functions on $(G / P)_{a}^{s f}$ are constants, so $f:(G / P)_{a}^{s f} \rightarrow Y$ is a universal torsor.

Proof. By Lemma 2.6 we need to show that Pic $\overline{\mathscr{T}}=0$ and $\bar{k}[\mathscr{T}]^{*}=\bar{k}^{*}$ where we write $\mathscr{T}=(G / P)_{a}^{s f}$ (see Section 1). The Picard group of $(G / P)_{a}$ is trivial since that of $G / P$ is generated by the class of a hyperplane section. Thus it suffices to show that the complement to $(G / P)_{a}^{s f}$ in $(G / P)_{a}$ has codimension at least 2 . The set of unstable points has codimension at least 2, by Proposition 2.4. The closed subset of its complement consisting of the stable points with nontrivial (finite) stabilizers in $T$ also has codimension at least 2, as follows from Corollary 2.3.

\section{Hermitian symmetric pairs}

Let $\mathfrak{g}$ be a semisimple Lie algebra over the field $k$ with Chevalley basis $\left\{H_{\beta}, X_{\gamma}\right\}$, where $\gamma$ is a root of $\mathrm{R}$, and $H_{\beta}=\left[X_{\beta}, X_{-\beta}\right]$, where $\beta$ is a simple root of $\mathrm{R}$.

A simple root $\alpha$ of $\mathfrak{g}$ defines a $\mathbf{Z}$-grading on $\mathfrak{g}$ in the following way. We set $\operatorname{deg}\left(X_{\alpha}\right)=1, \operatorname{deg}\left(X_{-\alpha}\right)=-1, \operatorname{deg}\left(X_{ \pm \beta}\right)=0$ for all other simple roots $\beta \neq \alpha$, and $\operatorname{deg}\left(H_{\beta}\right)=0$ for all simple roots $\beta$. Then

$$
\mathfrak{g}=\bigoplus_{i=-l(\alpha)}^{l(\alpha)} \mathfrak{g}_{i},
$$


where $l(\alpha)$ is the label of $\alpha$, that is, the coefficient of $\alpha$ in the decomposition of the maximal root as a linear combination of the simple roots. The Lie algebra $\mathfrak{p}=\bigoplus_{i \geq 0} \mathfrak{g}_{i}$ is the parabolic subalgebra defined by $\alpha$, and $\mathfrak{n}=\bigoplus_{i<0} \mathfrak{g}_{i}$ is the complementary nilpotent algebra. The center of the Lie algebra $\mathfrak{g}_{0}$ is one-dimensional, so $\mathfrak{g}_{0}=Z\left(\mathfrak{g}_{0}\right) \oplus \mathfrak{g}^{\prime}$, where $\mathfrak{g}^{\prime}$ is the semisimple Lie algebra whose Dynkin diagram is that of $\mathfrak{g}$ with the node corresponding to $\alpha$ removed.

It is clear from (4) that $l(\alpha)=1$ if and only if $[\mathfrak{n}, \mathfrak{n}]=0$. The following terminology has its origin in the theory of symmetric spaces; see [Helgason 2001, Chapter VIII].

Definition 3.1. The pair $(\mathrm{R}, \alpha)$ is a Hermitian symmetric pair if $l(\alpha)=1$, or, equivalently, if $\mathfrak{n}$ is a commutative Lie algebra.

If $\mathrm{R}$ is simply laced, then ( $\mathrm{R}, \alpha)$ is a Hermitian symmetric pair if and only if $\mathrm{R}=\mathrm{A}_{n}$, or if it is one of the following pairs: $\left(\mathrm{D}_{n}, \alpha_{i}\right)$, where $i=1, n-1$ or $n$, $\left(\mathrm{E}_{6}, \alpha_{1}\right),\left(\mathrm{E}_{6}, \alpha_{6}\right)$, and $\left(\mathrm{E}_{7}, \alpha_{7}\right)$.

We now assume that $\mathfrak{n}$ is commutative. Our next goal is to explore the implications of this assumption for the restriction of the $\mathfrak{g}$-module $V$ to the semisimple subalgebra $\mathfrak{g}^{\prime}$. We write $\mathrm{U}(\mathfrak{l})$ for the universal enveloping algebra of the Lie algebra $\mathfrak{l}$, and $S(W)$ for the symmetric algebra of the vector space $W$. Since $\mathfrak{n}$ is commutative we have $\mathrm{U}(\mathfrak{n})=S(\mathfrak{n})$.

The line $k v$ is a 1-dimensional $\mathfrak{p}$-submodule of $V$; hence the $\mathfrak{g}$-module $V$ is the quotient of the induced module $\mathrm{U}(\mathfrak{g}) \otimes_{\mathrm{U}(\mathfrak{p})} k v$ by the submodule generated by $X_{-\alpha}^{2} v$. (This follows from the construction of $V$ as the quotient of the Verma module by the submodule generated by $X_{-\beta} v$ for the simple roots $\beta \neq \alpha$, and $X_{-\alpha}^{2} v$.) By the Poincaré-Birkhoff-Witt theorem we have $\mathrm{U}(\mathfrak{g})=\mathrm{U}(\mathfrak{p}) \otimes_{k} \mathrm{U}(\mathfrak{n})$. The line $k v$ is a trivial $\mathfrak{g}^{\prime}$-module. Therefore, the $\mathfrak{g}^{\prime}$-module $\mathrm{U}(\mathfrak{g}) \otimes_{\mathrm{U}(\mathfrak{p})} k v$ is isomorphic to $\mathrm{U}(\mathfrak{n})=S(\mathfrak{n})$, so the finite dimensional vector space $V$ inherits the $\mathbf{Z}_{\leq 0}$-graded commutative $k$-algebra structure from $S(\mathfrak{n}), V=\bigoplus_{n \leq 0} V^{n}$. We turn this grading into a $\mathbf{Z}_{\geq 0}$-grading by setting $V_{n}=V^{-n}$. Since $\mathfrak{g}^{\prime}$ has grading 0 , the direct sum $V=\bigoplus_{n \geq 0} V_{n}$ is the direct sum of $\mathfrak{g}^{\prime}$-modules, and we can write

$$
V=k \oplus \mathfrak{n} \oplus\left(S^{\geq 2}(\mathfrak{n}) / S(\mathfrak{n}) \mathrm{U}\left(\mathfrak{g}^{\prime}\right) X_{-\alpha}^{2}\right),
$$

where $k=V_{0}, \mathfrak{n}=V_{1}$. Note that $1 \in V_{0}$ is a highest weight vector; it generates $V$ as a $S(\mathfrak{n})$-module.

Lemma 3.2. Let $(\mathrm{R}, \alpha)$ be a Hermitian symmetric pair. Then the adjoint representation of $\mathfrak{g}^{\prime}$ on $V_{1}=\mathfrak{n}=\mathfrak{g}_{-1}$ is the irreducible representation such that $X_{-\alpha}$ is a highest weight vector. If $\mathrm{R}$ is simply laced, then the highest weight $\omega^{\prime}$ of $V_{1}$ is the sum of the fundamental weights corresponding to the nodes of the Dynkin diagram of $\mathrm{R}$ adjacent to the node $\alpha$. 
Proof. We have $\left[X_{\beta}, X_{-\alpha}\right]=0$ for all simple roots $\beta \neq \alpha$, so $X_{-\alpha}$ is annihilated by the positive roots of $\mathfrak{g}^{\prime}$. Every root of $\mathfrak{n}$ is the sum of $-\alpha$ and a root of $\mathfrak{g}^{\prime}$, so $\mathfrak{n}$ is generated by $X_{-\alpha}$ as a $\mathfrak{g}^{\prime}$-module. The computation of the weight of $X_{-\alpha}$ is immediate from the defining relations among the elements of the Chevalley basis.

We have the exponential map

$$
\exp : \mathfrak{n} \rightarrow S(\mathfrak{n}), \quad \exp (u)=1+u+\frac{1}{2} u^{2}+\frac{1}{3 !} u^{3}+\cdots .
$$

Let $G$ be the simply connected semisimple algebraic $k$-group with Lie algebra $\mathfrak{g}$, $P \subset G$ the parabolic subgroup with Lie algebra $\mathfrak{p}$, and $N$ the unipotent $k$-group with Lie algebra $\mathfrak{n}$. By the Chevalley construction of the Lie group from its Lie algebra, $N$ acts on $V$ by the rule $1+x \mapsto \exp (x)$. Recall that the open Schubert cell of $G / P \subset \mathbb{P}(V)$ is the $N$-orbit of the highest weight vector, and hence is identified with $\exp (\mathfrak{n})$. (In particular, $\operatorname{dim} G / P=\operatorname{dim} V_{1}$.) Thus $\exp (x)$ is a polynomial $G^{\prime}$-equivariant map, where $G^{\prime}$ is the simply connected semisimple $k$-group with Lie algebra $\mathfrak{g}^{\prime}$

$$
\exp : V_{1} \rightarrow(G / P)_{a} \subset V=\bigoplus_{n \geq 0} V_{n} .
$$

Let $p: V_{1}=\mathfrak{n} \rightarrow V_{2}$ be the degree 2 graded component of $\exp (x)$.

Lemma 3.3. Let $G^{\prime}$ be the simply connected semisimple k-group with the Lie algebra $\mathfrak{g}^{\prime}$, and $P^{\prime} \subset G^{\prime}$ the parabolic subgroup which is the stabilizer of the line spanned by the highest weight vector $X_{-\alpha} \in \mathfrak{n}$. The restriction of $\exp (x)$ to $\left(G^{\prime} / P^{\prime}\right)_{a}$ coincides with $(1, \mathrm{id}, 0,0, \ldots)$. We have $\left(G^{\prime} / P^{\prime}\right)_{a}=p^{-1}(0)$, and the ideal of $\left(G^{\prime} / P^{\prime}\right)_{a}$ is generated by the coordinates of $p(x)$.

Proof. It is clear that every graded component of $\exp (x)$ of degree at least 2 sends the orbit $\left(G^{\prime} / P^{\prime}\right)_{a}$ of the highest weight vector $X_{-\alpha}$ to 0 . Indeed, $X_{-\alpha}^{m}$ is in the kernel of the natural map $S^{m}(\mathfrak{n}) \rightarrow V_{m}$, for $m \geq 2$. To prove the second statement let us observe that the symmetric square $S^{2}(\mathfrak{n})$ decomposes as the direct sum of $V_{2}$ and the $\mathfrak{g}^{\prime}$-submodule generated by $X_{-\alpha}^{2}$, which is the irreducible representation $V\left(2 \omega^{\prime}\right)$ with highest weight $2 \omega^{\prime}$. It is well known from [Lancaster and Towber 1979, proof of Theorem 1.1] or [Batyrev and Popov 2004, Proposition 4.2] that the orbit of the highest weight vector is the intersection of the second Veronese embedding with $V\left(2 \omega^{\prime}\right)$. This completes the proof.

Consider the following series of root systems,

$$
\mathrm{A}_{4} \subset \mathrm{D}_{5} \subset \mathrm{E}_{6} \subset \mathrm{E}_{7}
$$

Let $(\mathrm{R}, \alpha)$ be one of the Hermitian symmetric pairs

$$
\left(\mathrm{A}_{4}, \alpha_{3}\right), \quad\left(\mathrm{D}_{5}, \alpha_{5}\right), \quad\left(\mathrm{E}_{6}, \alpha_{6}\right), \quad\left(\mathrm{E}_{7}, \alpha_{7}\right),
$$


where the roots are numbered as in [Bourbaki 1981]. By Lemma 3.2 the pair $\left(G^{\prime}, P^{\prime}\right)$ is defined by $\left(\mathrm{R}^{\prime}, \alpha^{\prime}\right)$ which is the previous pair to $(\mathrm{R}, \alpha)$ in $(6)$. In other words, $P^{\prime}$ corresponds to the only node of the smaller diagram adjacent to $\alpha$. (If $G$ is of type $\mathrm{A}_{4}$, then $G^{\prime}$ is of type $\mathrm{A}_{1} \times \mathrm{A}_{2}, G^{\prime} / P^{\prime} \simeq \mathbb{P}^{1} \times \mathbb{P}^{2}$, but we shall not have to consider this case.)

We note that the fundamental weight $\omega$ dual to $\alpha$ is minuscule, that is, the weights of $V$ are $\mathrm{W} \omega$, and $\mathrm{W} v$ is a basis of $V$; see [Bourbaki 1981, VIII.7.3]. We also note that the $G$-module $V$ defined by $\omega$ is faithful (this follows from the fact that $\omega$ generates $P(\mathrm{R}) / Q(\mathrm{R})$, which can be checked from the tables). Thus the faithful representation of $G$ in $V$ defines a faithful representation of $G^{\prime}$, and this implies that $G^{\prime} \subset G$ (in fact, $G^{\prime}$ is the Levi subgroup of $P$ ).

Let us identify the graded components of $V$ in various cases. Let $d_{r}=\operatorname{dim} V$. We have

$$
d_{4}=10, d_{5}=16, d_{6}=27, d_{7}=56 .
$$

The details given below show that for $r=4,5,6$ the graded components of $\exp (x)$ of degree at least 3 are zero.

Let $\mathrm{R}=\mathrm{A}_{4}$. Then $G=\mathrm{SL}(5)$, and $G / P$ is the Grassmannian $G(2,5)$. Denote by $E_{n}$ the standard $n$-dimensional representation of $\operatorname{SL}(n)$. We have $V=\Lambda^{2}\left(E_{5}\right)$, $\operatorname{dim} V=10=1+6+3$. The group $G^{\prime}=\operatorname{SL}(2) \times \operatorname{SL}(3)$ is embedded into $\operatorname{SL}(5)$ in the obvious way, and the graded factors of $V$ are $V_{1}=E_{2} \otimes E_{3}, V_{2}=\Lambda^{2}\left(E_{3}\right) \cong E_{3}^{*}$. The map $p: V_{1} \rightarrow V_{2}$ sends $x$ to the $\Lambda^{2}\left(E_{3}\right)$-component of

$$
x \wedge x \in \Lambda^{2}\left(E_{5}\right)=\Lambda^{2}\left(E_{2}\right) \oplus\left(E_{2} \otimes E_{3}\right) \oplus \Lambda^{2}\left(E_{3}\right) .
$$

Let $\mathrm{R}=\mathrm{D}_{5}$. Then $V$ is a spinor representation of $G=\operatorname{Spin}(10)$ of dimension $16=1+10+5$, and $G / P$ is the isotropic Grassmannian (one of two families of maximal isotropic subspaces of the nondegenerate quadric of rank 10), and $\operatorname{dim} G / P=10$. The graded components are $V_{1}=\Lambda^{2}\left(E_{5}\right)$ and $V_{2}=\Lambda^{4}\left(E_{5}\right) \cong E_{5}^{*}$. The map $p: V_{1} \rightarrow V_{2}$ sends $x$ to $x \wedge x$.

Let $\mathrm{R}=\mathrm{E}_{6}$. Then $\operatorname{dim} V=27=1+16+10, V_{1}$ is the spinor representation of $\operatorname{Spin}(10)$ as above, and $V_{2}$ is the standard 10-dimensional representation of $\mathrm{SO}(10)$. We have $\operatorname{dim} G / P=16$.

Let $\mathrm{R}=\mathrm{E}_{7}$. Then $\operatorname{dim} V=56=1+27+27+1, V_{1}$ is the 27-dimensional representation of the group of type $\mathrm{E}_{6}$ considered above, $V_{2}=\left(V_{1}\right)^{*}$, and $V_{3}=k$ is the trivial 1-dimensional representation. (The graded components of degree at least 4 are zero.) We have $\operatorname{dim} G / P=27$. We define $q: V_{1}=\mathfrak{n} \rightarrow V_{3}=k$ as the degree 3 graded component of $\exp (x)$. This is a $\mathrm{E}_{6}$-invariant cubic form in 27 variables. The 27 weight coordinates of $p(x)$ are partial derivatives of $q(x)$. This identifies the space $G / P$ of type $\mathrm{E}_{6}$ with the singular locus of the cubic hypersurface $q(x)=0$. 
Define a symmetric bilinear form $p(x, y)$ on $V_{1}$ with values in $V_{2}$ by the formula $p(x+y)=p(x)+2 p(x, y)+p(y)$. Then $\exp (x+y)=\exp (x) \exp (y)$ implies that

$$
2 p(x, y)=x \cdot y
$$

is the product of $x \in V_{1}$ and $y \in V_{1}$ in the commutative $k$-algebra $V$.

We have a decomposition of $S^{2}\left(V_{1}\right)$ as the direct sum of $V_{2}$ and the representation with highest weight $2 \omega^{\prime}$ (see the proof of Lemma 3.3). In the notation of [Bourbaki 1981] the representation $V_{2}$ is irreducible with highest weight $\omega_{1}$; in particular, it is minuscule. Thus the eigenspaces for the action of the maximal torus $H^{\prime}=H \cap G^{\prime}$ are 1-dimensional, so on $V_{2}$, in the same way as on $V_{1}$, we have weight coordinates well defined up to a multiplicative constant. The coordinates $p_{\lambda}(x, y)$ of $p(x, y)$ are symmetric bilinear forms of degree 2 with values in $k$. We can write

$$
p_{\lambda}(x, y)=\sum_{\lambda=\mu+\nu} p_{\mu \nu} x_{\mu} y_{\nu}
$$

where $\mu$ and $v$ are weights of $V_{1}, p_{\mu \nu} \in k$, and $x_{\mu}$ is a nonzero linear form on the weight $\mu$ subspace $\left(V_{1}\right)_{\mu} \subset V_{1}$ (and similarly for $y_{\nu}$ ). One checks that for $r=4,5,6,7$ the ranks of the quadratic forms $p_{\lambda}(x)$ are $4,6,8,10$, respectively. If $r=7$ we associate to the cubic form

$$
q(x)=\sum_{\mu+\nu+\xi=0} q_{\mu \nu \xi} x_{\mu} x_{\nu} x_{\xi}
$$

the symmetric trilinear form

$$
q(x, y, z)=\sum_{\mu+\nu+\xi=0} q_{\mu \nu \xi} x_{\mu} y_{\nu} z_{\xi} .
$$

In this case the weights of $V_{2}$ are the negatives of the weights of $V_{1}$. Moreover, $p_{-\mu}(x)=\partial q(x) / \partial x_{\mu}$, so

$$
\begin{gathered}
3 q(x, y, z)=\sum_{\mu} p_{-\mu}(x, y) z_{\mu}, \\
p_{-\mu}(x, y)=\sum_{-\mu=\nu+\xi} 3 q_{\mu \nu \xi} x_{\nu} y_{\xi} .
\end{gathered}
$$

For future reference we note that if $p_{\lambda}(x, y)=0$ for all $\lambda$, then $q(x, y, y)=0$. It follows from $\exp (x+y)=\exp (x) \exp (y)$ that

$$
3 q(x, x, y)=p(x) \cdot y
$$

is the product of $p(x) \in V_{2}$ and $y \in V_{1}$ in the commutative $k$-algebra $V$. 


\section{4. $G / P$ and blowing-up}

Let $\pi:(G / P)_{a} \rightarrow V_{1}$ be the restriction to $(G / P)_{a}$ of the natural projection

$$
V=k \oplus V_{1} \oplus V_{2} \oplus V_{3} \rightarrow V_{1}
$$

We have $\exp (x)=(1, x, p(x), q(x))$; hence $\pi \circ \exp =\mathrm{id}$. Here and in what follows we write our formulae for the case $r=7$, with the convention that if $r<7$ the last coordinate must be discarded.

We now describe the fibres of $\pi$.

Lemma 4.1. Let $g_{t}=\left(t, 1, t^{-1}, t^{-2}\right), t \in \bar{k}^{*}$. For $x \in V_{1} \otimes_{k} \bar{k}$ we have the following statements.

(a) If $x \notin\left(G^{\prime} / P^{\prime}\right)_{a}$, then $\pi^{-1}(x)=\left\{g_{t} \cdot \exp (x) \mid t \in \bar{k}^{*}\right\}$.

(b) If $x \in\left(G^{\prime} / P^{\prime}\right)_{a} \backslash\{0\}$, then

$$
\pi^{-1}(x)=\left\{(t, x, 0,0) \mid t \in \bar{k}^{*}\right\} \cup\left\{\left(0, x, 2 p_{\lambda}(x, u), 3 q(x, u, u)\right) \mid u \in V_{1} \otimes_{k} \bar{k}\right\} .
$$

Proof. Recall that the torus $T$ is generated by the maximal torus $H \subset G$ and the scalar matrices $(t, t, t, t), t \in \bar{k}^{*}$. Let $h \in \mathfrak{h}$ be an element of the Lie algebra of $H$ such that $\beta(h)=0$ for all simple roots $\beta$ of $G, \beta \neq \alpha$, and $\alpha(h)=1$. The 1-parameter subgroup $\mathbf{G}_{m} \subset H$ whose tangent vector at the identity is $h$, acts on $V$ as $\left(t^{m}, t^{m-1}, t^{m-2}, t^{m-3}\right)$, where $m=\omega(h)$, and $\omega$ is the fundamental weight dual to $\alpha$. Hence $g_{t} \in T$ for any $t \in \bar{k}^{*}$.

Every $\bar{k}$-point $y=\left(y_{0}, y_{1}, y_{2}, y_{3}\right)$ of the closed set $(G / P)_{a}$ satisfies the equations

$$
y_{0} y_{2}=p\left(y_{1}\right), \quad y_{0}^{2} y_{3}=q\left(y_{1}\right),
$$

since these are satisfied on the affine cone over $\exp \left(V_{1}\right)$ which is dense in $(G / P)_{a}$. Therefore, if $\pi$ sends a $\bar{k}$-point $y$ of $(G / P)_{a}$ to $x=y_{1}$, and $y_{0} \neq 0$, we can write $y=g_{t} \cdot(1, x, p(x), q(x))=g_{t} \cdot \exp (x)$ for $t=y_{0} \in \bar{k}^{*}$. All such points are in $(G / P)_{a}$ since the action of $T$ preserves $(G / P)_{a}$, and $\exp \left(V_{1}\right) \subset(G / P)_{a}$. If $y_{0}=0$ we see from (11) and Lemma 3.3 that $x \in\left(G^{\prime} / P^{\prime}\right)_{a}$. This proves (a).

To prove (b), assume $x \in\left(G^{\prime} / P^{\prime}\right)_{a}, x \neq 0$. If $y_{0} \neq 0$, then $y=(t, x, 0,0)$, by (11).

We need some preparations for the case $y_{0}=0$. Recall that $V_{0}$ is identified with $k$ by the choice of a highest weight vector $v \in V_{0}$, and $V_{1}$ is identified with $\mathfrak{n}$. Consider $\mathfrak{g}_{1}=\mathfrak{n}^{-}$, the opposite nilpotent algebra of $\mathfrak{n}$. Any nonzero element $X \in \mathfrak{g}_{1}$ sends $V_{i}$ to $V_{i-1}$ because of the grading. Hence we can write $\exp (X t)\left(y_{0}, y_{1}, y_{2}, y_{3}\right)=\left(y_{0}+s\left(y_{1}, X\right) t+z_{1} t^{2}+z_{2} t^{3}, y_{1}+u_{1} t+u_{2} t^{2}, y_{2}+w t, y_{3}\right)$, where $z_{1}, z_{2} \in k, u_{1}, u_{2} \in V_{1}, w \in V_{2}$, and $s\left(y_{1}, X\right) \in k$ is defined by

$$
s\left(y_{1}, X\right) v=X y_{1} v=\left[X, y_{1}\right] v .
$$


For any nonzero $y_{1} \in \mathfrak{n} \otimes_{k} \bar{k}=V_{1} \otimes_{k} \bar{k}$ one can find $X \in \mathfrak{g}_{1} \otimes_{k} \bar{k}$ such that $s\left(y_{1}, X\right)=1$. Otherwise $\mathfrak{g}_{1} y_{1} v=0$, and so $y_{1} v$ is a highest vector of the $\mathfrak{g}$-module $V \otimes_{k} \bar{k}$, which is not a multiple of $v$. This contradicts the irreducibility of $V \otimes_{k} \bar{k}$. Fix such an element $X \in \mathfrak{g}_{1} \otimes_{k} \bar{k}$.

Now let $y_{0}=0$. Then

$$
g_{t^{-1}} \exp (X t)\left(0, y_{1}, y_{2}, y_{3}\right)=\left(1+z_{1} t+z_{2} t^{2}, y_{1}+u_{1} t+u_{2} t^{2}, y_{2} t+w t^{2}, y_{3} t^{2}\right)
$$

is a $\bar{k}[t]$-point of $(G / P)_{a}$, and hence its coordinates satisfy (11) identically in $t$. Equating to 0 the coefficient at $t$ in the first equation in (11) we obtain $y_{2}=$ $2 p\left(y_{1}, u\right)$, where $u=u_{1}$. Equating to 0 the coefficient at $t^{2}$ in the second equation, and using that $q\left(y_{1}, y_{1}, v\right)=0$ for all $v \in V_{1}$ according to (9), we obtain $y_{3}=$ $3 q\left(y_{1}, u, u\right)$.

To complete the proof of (b) we need to show that for any $\bar{k}$-point $x \in\left(G^{\prime} / P^{\prime}\right)_{a}$ and any $u \in V_{1} \otimes_{k} \bar{k}$ the point $\left(0, x, 2 p_{\lambda}(x, u), 3 q(x, u, u)\right)$ is contained in $(G / P)_{a}$. We note that

$$
\left(0, x, 2 p_{\lambda}(x, u), 3 q(x, u, u)\right)=\exp (u) \cdot(0, x, 0,0),
$$

as immediately follows from (7) and (10). Since $\exp (u)$ is in the unipotent group $N \subset G$ it is enough to show that $(0, x, 0,0)$ is in $(G / P)_{a}$. Clearly $(1, x, 0,0)=$ $\exp (x)$ is in $(G / P)_{a}$. Choosing $X \in \mathfrak{g}_{1} \otimes_{k} \bar{k}$ as above such that $s(x, X)=-1$ we obtain $\exp (X)(1, x, 0,0)=(0, x, 0,0)$.

Corollary 4.2. Let $U \subset(G / P)_{a}$ be the complement to the intersection of $(G / P)_{a}$ with $\left(V_{0} \oplus V_{1}\right) \cup\left(V_{2} \oplus V_{3}\right)$. The restriction of $\pi$ to $U$ is a morphism $U \rightarrow V_{1} \backslash\{0\}$, which is the composition of a torsor under the torus $\mathbf{G}_{m}=\left\{g_{t} \mid t \in \bar{k}^{*}\right\}$, and the morphism inverse to the blowing-up of $V_{1} \backslash\{0\}$ at $\left(G^{\prime} / P^{\prime}\right)_{a} \backslash\{0\}$.

Proof. The set $U$ is covered by the open subsets $U_{0}: y_{0} \neq 0$, and $U_{\lambda}: y_{\lambda} \neq 0$, where $y_{\lambda}$ are the weight coordinates in $V_{2}$. Indeed, if $y_{0}=y_{\lambda}=0$ for all $\lambda$, then we are in case (b) of Lemma 4.1, but $p_{\lambda}(x, u)=0$ for all $\lambda$ implies $q(x, u, u)=0$, and such points are not in $U$. Each of these open subsets is $\mathbf{G}_{m}$-equivariantly isomorphic to the direct product of $\mathbf{G}_{m}$ and the closed subvariety of $(G / P)_{a}$ given by $y_{i}=1$ with trivial $\mathbf{G}_{m}$-action. Gluing them together we obtain the quotient $\tilde{U}$.

The equations (11) show that $\pi^{-1}(0) \cap U=\varnothing$; thus $\pi$ projects $U$ to $V_{1} \backslash\{0\}$. The action of $\mathbf{G}_{m}$ preserves the fibres, hence $\pi$ factors through a morphism $\tilde{U} \rightarrow V_{1} \backslash\{0\}$. It is an isomorphism outside $\left(G^{\prime} / P^{\prime}\right)_{a}$, whereas the inverse image of $\left(G^{\prime} / P^{\prime}\right)_{a} \backslash\{0\}$ is the projectivisation of the normal bundle to $\left(G^{\prime} / P^{\prime}\right)_{a} \backslash\{0\}$ in $V_{1} \backslash\{0\}$, by Lemma 4.1 (b). It is not hard to prove (and is well known to experts) that this implies that $\tilde{U}$ is the blowing-up of $V_{1} \backslash\{0\}$ at $\left(G^{\prime} / P^{\prime}\right)_{a} \backslash\{0\}$. 


\section{Del Pezzo surfaces}

For the geometry of exceptional curves on del Pezzo surfaces the reader is referred to [Manin 1986, Chapter IV]; see also [Friedman and Morgan 2002, Section 5]. Let $M_{1}, \ldots, M_{r}, 4 \leq r \leq 7$, be $k$-points in general position in the projective plane $\mathbb{P}^{2}$, which says that no three points are on a line and no six on a conic. The blowing-up $X$ of $\mathbb{P}^{2}$ in $M_{1}, \ldots, M_{r}$ is called a split del Pezzo surface of degree $d=9-r$. The surface $X$ contains exactly $d_{r}$ exceptional curves, that is, smooth rational curves with self-intersection -1 . For $r \leq 6$ the exceptional curves on $X$ arise in one of these ways: the inverse images of the $M_{i}$; the proper transforms of the lines through $M_{i}$ and $M_{j}, i \neq j$; the proper transforms of the conics through five of the $M_{i}$. For $r=7$ one also has the proper transforms of singular cubics passing through all 7 points with a double point at some $M_{i}$. The intersection index defines an integral bilinear form (.) on Pic $X$. The opposite of the canonical class $-K_{X}$ is an ample divisor, $\left(K_{X}^{2}\right)=d$. The Picard group Pic $\bar{X}=\operatorname{Pic} X$ is generated by the classes of exceptional curves (the complement to the union of these curves is an open subset of $\mathbb{A}^{2}$ ). The triple (Pic $X, K_{X},($.$) ) coincides, up to isomorphism, with the triple$ $\left(N_{r}, K_{r},().\right)$ defined as [Manin 1986, Theorem 23.9]

$N_{r}=\bigoplus_{i=0}^{r} \mathbf{Z} \ell_{i}, \quad K_{r}=-3 \ell_{0}+\sum_{i=1}^{r} \ell_{i}, \quad\left(\ell_{0}^{2}\right)=1,\left(\ell_{i}^{2}\right)=-1, i \geq 1,\left(\ell_{i} \cdot \ell_{j}\right)=0, i \neq j$

Moreover, the exceptional curves are identified with the elements $\ell \in N_{r}$ such that $\left(\ell^{2}\right)=\left(\ell . K_{r}\right)=-1$, which are called the exceptional classes [Manin 1986, Theorem 23.8]. By definition, a geometrically integral conic on $X$ is a smooth rational curve with self-intersection 0. By the Riemann-Roch theorem each conic belongs to a 1-dimensional pencil of curves which are fibres of a morphism $X \rightarrow \mathbb{P}^{1}$, called a conic bundle. We refer to the fibres of such a morphism as conics. In particular, through every point of $X$ passes exactly one conic of a given pencil. The classes of conic bundles can be characterized by the properties $\left(c^{2}\right)=0,\left(c . K_{r}\right)=-2$.

Let $K_{r}^{\perp}$ be the orthogonal complement to $K_{r}$ in $N_{r}$. The elements $\alpha \in K_{r}^{\perp}$ such that $\left(\alpha^{2}\right)=-2$ form a root system $\mathrm{R}$ in the vector space $K_{r}^{\perp} \otimes \mathbb{R} \simeq \mathbb{R}^{r}$ with the negative definite scalar product (.). In fact, $\mathrm{R}$ is a root system of rank $r$ in the series (5). Moreover, the lattice $K_{r}^{\perp}$ is generated by roots so $K_{r}^{\perp} \simeq Q(\mathrm{R})$. For example, we can choose

$$
\beta_{1}=-\ell_{1}+\ell_{2}, \ldots, \beta_{r-1}=-\ell_{r-1}+\ell_{r}, \beta_{r}=-\ell_{0}+\left(\ell_{1}+\ell_{2}+\ell_{3}\right)
$$

as a basis of simple roots of $\mathrm{R}$. The relation to our standard numeration, which follows [Bourbaki 1981], is $\alpha_{r}=\beta_{r-1}, \alpha_{1}=\beta_{1}$.

The Weyl group $\mathrm{W}=\mathrm{W}(\mathrm{R})$ generated by the reflections in the roots, is the automorphism group of the triple $\left(N_{r}, K_{r},().\right)$. It operates transitively on the set 
of exceptional curves, and also on the set of conic bundle classes; see, for example, [Friedman and Morgan 2002, Lemma 5.3]. Let

$$
P(\mathrm{R})=\left\{n \in K_{r}^{\perp} \otimes \mathbb{R}:(n . m) \in \mathbf{Z} \text { for any } m \in Q(\mathrm{R})\right\}
$$

be the lattice dual to $Q(\mathrm{R})$; we have $Q(\mathrm{R}) \subset P(\mathrm{R})$. The image of the map

$$
N_{r} \rightarrow N_{r} \otimes \mathbb{R}=\mathbb{R} K_{r} \oplus\left(K_{r}^{\perp} \otimes \mathbb{R}\right)
$$

is contained in the orthogonal direct $\operatorname{sum} \frac{1}{d} \mathbf{Z} K_{r} \oplus P(\mathrm{R})$ as a subgroup of index $d$.

Lemma 5.1. Let $\alpha=\beta_{r-1} \in \mathrm{R}$ be the simple root such that $(\mathrm{R}, \alpha)$ is one of the pairs in (6), and let $\omega \in P(\mathrm{R})$ be the dual fundamental weight, $(\alpha . \omega)=-1$.

(i) The exceptional classes in $N_{r}$ are $-\frac{1}{d} K_{r}+w \omega$, for all $w \in \mathrm{W}$.

(ii) Two distinct exceptional curves intersect in $X$ if and only if the corresponding weights are not adjacent vertices of the convex hull $\operatorname{Conv}(\mathrm{W} \omega)$.

(iii) Let $\omega_{1}$ be the fundamental weight dual to the root $\beta_{1}$. The conic bundle classes in $N_{r}$ are $-\frac{2}{d} K_{r}+w \omega_{1}$, for all $w \in \mathrm{W}$.

Note that since $\mathrm{W}$ acts transitively on the set of bases, the choice of a basis of simple roots is not important for the conclusion of this lemma.

Proof. (i) and (iii) The image of the exceptional class $\ell_{r}$ in $P(\mathrm{R})$ is the fundamental weight $\omega=\omega_{r-1}$, and the image of the conic bundle class $\ell_{0}-\ell_{1}$ is the fundamental weight $\omega_{1}$. The statement now follows from the transitivity of action of $\mathrm{W}$ on these classes. See [Friedman and Morgan 2002, Lemma 5.2].

(ii) By the transitivity of $\mathrm{W}$ on the exceptional classes it is enough to check this for the classes $-\frac{1}{d} K_{r}+\omega$ and $-\frac{1}{d} K_{r}+x$, where $x=w \omega$ for some $w \in \mathrm{W}$. The intersection index

$$
\left(-\frac{1}{d} K_{r}+x .-\frac{1}{d} K_{r}+\omega\right)=\frac{1}{d}+(x . \omega)
$$

equals $-L(x)$ in the notation of the proof of Proposition 2.4 (with the opposite sign of the scalar product). In the simply laced case this proof shows that $L(x)=1$ when $x=\omega, L(x)=0$ if $x$ is a vertex of the convex hull Conv $(\mathrm{W} \omega)$ adjacent to $\omega$, and $L(x)<0$ for all other $x \in \mathrm{W} \omega$.

We observe that for any conic bundle class $x$ there exists a conic bundle class $y$ such that $(x \cdot y)=1$. Indeed, by the transitivity of $\mathrm{W}$ on conic bundle classes we can assume that $x=\ell_{0}-\ell_{1}$. For $y=\ell_{0}-\ell_{2}$ we have $(x \cdot y)=1$.

\section{Main theorem}

We recall our notation.

- $(\mathrm{R}, \alpha)$ is the pair in (6) such that $\mathrm{R}$ has rank $r$; 
- $G$ is the simply connected semisimple group with a split maximal torus $H$ and a maximal parabolic subgroup $P \supset H$, such that $(G, P)$ is defined by the pair $(\mathrm{R}, \alpha)$;

- $V$ is the fundamental representation of $G$ such that $P$ is the stabilizer of the line spanned by a highest weight vector (this representation is faithful);

- $T \subset \mathrm{GL}(V)$ is the torus generated by the image of $H$ in $\mathrm{GL}(V)$, and the scalar matrices;

- $Y$ is the geometric quotient of $(G / P)_{a}^{s f} \subset(G / P)_{a}$ with respect to the natural left action of $T$;

- the morphism $f:(G / P)_{a}^{s f} \rightarrow Y$ is a universal torsor (see Theorem 2.7).

Let $\Lambda \subset \hat{H}$ be the set of weights of $H$ in $V$, and let $V_{\lambda} \subset V$ be the subspace of weight $\lambda$, so that $V=\bigoplus_{\lambda \in \Lambda} V_{\lambda}$. In our case $\operatorname{dim} V_{\lambda}=1$ (since $V$ is minuscule; see Section 3). Let $\pi_{\lambda}: V \rightarrow V_{\lambda}$ be the natural projections, and let $L_{\lambda}=\pi_{\lambda}^{-1}(0)$ be the weight coordinate hyperplanes. For a subset $A \subset V$ we write $A^{\times}$for the set of points of $A$ outside $\cup_{\lambda \in \Lambda} L_{\lambda}$. For a subset $B \subset Y$ we write $B^{\times}$for $f\left(f^{-1}(B)^{\times}\right)$.

We now state our main theorem, whose proof occupies the rest of the paper.

Theorem 6.1. For $r=4,5,6$ or 7 let $M_{1}, \ldots, M_{r}$ be $k$-points in general position in $\mathbb{P}^{2}$ (no three on a line, no six on a conic). Let $X$ be the blowing-up of $\mathbb{P}^{2}$ in $M_{1}, \ldots, M_{r}$. There exists an embedding $X \hookrightarrow Y$ such that $X \backslash X^{\times}$is the union of exceptional curves on $X$. For such an embedding $f^{-1}(X) \rightarrow X$ is a universal torsor.

We write $S_{\chi}^{n}(V)$ for the $H$-eigenspace of $S^{n}(V)$ of weight $\chi \in \hat{H}$, and $S_{\chi}^{n}(V)^{*}$ for the dual space of functions. Let $I(\mathscr{T}) \subset k[V]=S\left(V^{*}\right)$ be the ideal of $\mathscr{T}$. We shall prove the following statement from which the main theorem will follow.

There exists an embedding of a universal torsor $\mathcal{T}$ over $X$ into $(G / P)_{a}^{s f} \subset V$ such that the restriction of $f$ to $\mathscr{T}$ is the structure morphism $\mathscr{T} \rightarrow X$, and $f\left(\mathscr{T}^{\times}\right)$is the complement to the union of exceptional curves on X. Moreover, for $r<7$ the ideal $I\left(\mathscr{T}^{\times}\right) \subset k\left[V^{\times}\right]$is generated by the graded components of degree 2 and weight $w \omega_{1}$, for all $w \in \mathrm{W}$.

The last statement will be used in the case $r=7$, and can be ignored by the reader interested in the cases $r=5$ and $r=6$ only. Recall that $\omega_{1}$ is the highest weight of a nontrivial irreducible $\mathfrak{g}$-module of least dimension.

Proof. The proof is by induction on $r$ starting from $r=4$. In this case $Y$ is a del Pezzo surface of degree $5, G / P$ is the Grassmannian variety $G(3,5) \simeq G(2,5)$, and $G(3,5)^{s f}=G(3,5)^{s}$ is a universal torsor over $Y$; see [Skorobogatov 1993] or [Skorobogatov 2001, Lemma 3.1.6]. It is well known that the ideal of $G(3,5)_{a} \subset V$ 
is generated by the (quadratic) Plücker relations, and it is easy to see that their weights are of the form $w \omega_{1}$, so our statement is true in this case.

Suppose we know the statement for $r-1 \geq 4$. This means that we are given the following data.

- $\left(\mathrm{R}^{\prime}, \alpha^{\prime}\right)$ is the "previous" pair to $(\mathrm{R}, \alpha)$ in (6);

- $\mathrm{W}^{\prime}=\mathrm{W}\left(\mathrm{R}^{\prime}\right)$ is the Weyl group;

- $G^{\prime}$ and $P^{\prime}$ are defined by $\left(\mathrm{R}^{\prime}, \alpha^{\prime}\right)$, so that $\left(G^{\prime} / P^{\prime}\right)_{a} \subset V_{1}$ (see Section 3);

- $H^{\prime}=H \cap G^{\prime}$, so that $\mathrm{R}^{\prime}$ is the root system of $G^{\prime}$ with respect to $H^{\prime}$;

- $T^{\prime} \subset \mathrm{GL}\left(V_{1}\right)$ is the torus generated by the image of $H^{\prime}$ in $\mathrm{GL}\left(V_{1}\right)$ and the scalars ( $T^{\prime}$ is also the image of $H$ in $\left.\operatorname{GL}\left(V_{1}\right)\right)$;

- $x_{\mu}$ is a nonzero linear form on the weight $\mu$ subspace of $V_{1}$;

- $Y^{\prime}$ is the quotient of $\left(G^{\prime} / P^{\prime}\right)_{a}^{s f}$ by $T^{\prime}$;

- $f^{\prime}:\left(G^{\prime} / P^{\prime}\right)_{a}^{s f} \rightarrow Y^{\prime}$ is a universal torsor;

- $X^{\prime}$ is the blowing-up of $\mathbb{P}^{2}$ in $M_{1}, \ldots, M_{r-1}$ (it is a del Pezzo surface of degree $d^{\prime}=8-r$ );

- there exists an embedding $X^{\prime} \hookrightarrow Y^{\prime}$ satisfying the conditions of the theorem, in particular,

- $\mathscr{T}^{\prime}=f^{\prime-1}\left(X^{\prime}\right) \rightarrow X^{\prime}$ is a universal torsor.

The general position assumption implies that $M_{r}$ does not belong to the exceptional curves of $X^{\prime}$. Thus, by Hilbert's Theorem 90, we can find a $k$-point $x_{0} \in \mathscr{T}^{\prime \times}$ such that $f^{\prime}\left(x_{0}\right)=M_{r}$.

Let $\tau: \hat{T}^{\prime} \rightarrow$ Pic $X^{\prime}$ be the map defined in Section 1; up to the sign, $\tau$ coincides with the type of the torsor $f^{\prime}: \mathscr{T}^{\prime} \rightarrow X^{\prime}$ (Lemma 1.2). Since the torsor $f^{\prime}: \mathscr{T}^{\prime} \rightarrow X^{\prime}$ is universal, $\tau$ is an isomorphism of $\hat{T}^{\prime}=K\left[\mathscr{T}_{K}^{\prime}\right]^{*} / K^{*}$ and Pic $X^{\prime}$ as abelian groups. To account for the duality between vectors and linear forms on $V_{1}$ we identify these groups by the isomorphism $-\tau$. Recall that the Weyl group $\mathrm{W}^{\prime}$ acts on $\hat{T}^{\prime}$ via the normalizer of $H^{\prime}$ in $G^{\prime}$, permuting the weights of $V_{1}$. By induction assumption $-\tau$ sends these weights bijectively onto the exceptional classes in Pic $X^{\prime}$. If we transport the action of $\mathrm{W}^{\prime}$ from $\hat{T}^{\prime}$ to Pic $X^{\prime}$ using $-\tau$, then the action of $\mathrm{W}^{\prime}$ so obtained preserves the intersection index of exceptional curves; see (12). Thus $-\tau$ is a homomorphism of $\mathrm{W}^{\prime}$-modules, where $\mathrm{W}^{\prime}$ acts on Pic $X^{\prime}$ as the automorphism group of the triple $\left(N_{r-1}, K_{r-1},().\right)$. In particular, $-\tau$ identifies the $\mathrm{W}^{\prime}$-(co)invariants on both sides (isomorphic to $\mathbf{Z}$ ). This implies that if $\chi$ is a weight of $T^{\prime}$ in $S^{n}\left(V_{1}\right)$, then the restriction of $\chi$ to the scalar matrices $\mathbf{G}_{m} \subset T^{\prime}$ coincides with the intersection index of $-\tau(\chi)$ and $-K_{X^{\prime}}$, that is,

$$
\left(\tau(\chi) \cdot K_{X^{\prime}}\right)=n
$$


(the sign is uniquely determined by the fact that effective divisors intersect positively with $-K_{X^{\prime}}$ ). The isomorphism $-\tau$ also identifies the quotients by the $\mathrm{W}^{\prime}-$ invariants, that is, $P\left(\mathrm{R}^{\prime}\right)$ and $\hat{H}^{\prime}$. We fix these identifications from now on.

For $\phi(x) \in S_{\chi}^{n}\left(V_{1}\right)^{*}, \chi \in \hat{T}^{\prime}$, let $C_{\phi} \subset X^{\prime}$ be the image of the intersection of $\mathscr{T}^{\prime}$ with the $T^{\prime}$-invariant hypersurface $\phi(x)=0$. If $C_{\phi} \neq X^{\prime}$, then the class $\left[C_{\phi}\right]$ in Pic $X^{\prime}$ is $-\tau(\chi)$, and (13) can be written as

$$
\left(\left[C_{\phi}\right] \cdot\left(-K_{X^{\prime}}\right)\right)=n .
$$

We have (see the end of Section 1 for the first equality)

$$
\mathrm{H}^{0}\left(X^{\prime}, \mathcal{O}_{-\chi}\right)=k\left[\mathscr{T}^{\prime}\right]_{-\chi}=S_{\chi}^{n}\left(V_{1}\right)^{*} / I\left(\mathscr{T}^{\prime}\right) \cap S_{\chi}^{n}\left(V_{1}\right)^{*} .
$$

Apart from the weights of $V_{1}$ which correspond to the exceptional curves, the following two cases will be particularly relevant. For $n=2$ let $\lambda$ be a weight of $T^{\prime}$ in $V_{2}$. The restriction of $\lambda$ to $H^{\prime}$ is $w \omega_{1} \in \hat{H}^{\prime}=P\left(\mathrm{R}^{\prime}\right)$, where $w \in \mathrm{W}^{\prime}$ (see the end of Section 3). If $\phi \in S_{\lambda}^{2}\left(V_{1}\right)^{*}$ is such that $C_{\phi} \neq X^{\prime}$, then by (14) we see that $\left[C_{\phi}\right]=-\frac{2}{d^{\prime}} K_{X^{\prime}}+w \omega_{1}$, so $C_{\phi}$ is a conic on $X^{\prime}$ by Lemma 5.1 (iii). The Riemann-Roch theorem implies that $\operatorname{dim} \mathrm{H}^{0}\left(X^{\prime}, \mathrm{O}_{-\lambda}\right)=2$, where $\mathrm{O}_{-\lambda}=\mathcal{O}\left(C_{\phi}\right)$ is the invertible sheaf associated to $C_{\phi}$. Thus $I\left(\mathscr{T}^{\prime}\right) \cap S_{\lambda}^{2}\left(V_{1}\right)^{*}$ has codimension 2 in $S_{\lambda}^{2}\left(V_{1}\right)^{*}$. Note that by Lemma 3.3 we have $p_{\lambda}(x) \in I\left(\mathscr{T}^{\prime}\right) \cap S_{\lambda}^{2}\left(V_{1}\right)^{*}$. For $r=7$ and $n=3$ the space $V_{3}$ is a trivial 1-dimensional representation of $G^{\prime}$, hence of weight $0 \in \hat{H}^{\prime}$. Thus for $\phi \in S_{0}^{3}\left(V_{1}\right)^{*}$ we have $\left[C_{\phi}\right]=-K_{X^{\prime}}$, by (14). If $C_{\phi} \neq X^{\prime}$, then $C_{\phi}$ is a plane section of the cubic surface $X^{\prime} \subset \mathbb{P}^{3}$. The vector space $\mathrm{H}^{0}\left(X^{\prime}, \mathcal{O}\left(C_{\phi}\right)\right)=\mathrm{H}^{0}\left(X^{\prime}, \mathcal{O}\left(-K_{X^{\prime}}\right)\right)$ has dimension 4; thus $I_{0}=I\left(\mathscr{T}^{\prime}\right) \cap S_{0}^{3}\left(V_{1}\right)^{*}$ has codimension 4 in $S_{0}^{3}\left(V_{1}\right)^{*}$. It is clear that $q(x) \in I_{0}$; see, for example, (10).

The following proposition is a crucial technical step in the proof of our main theorem.

Proposition 6.2. There exists a nonempty open subset $\Omega\left(x_{0}\right) \subset\left(G^{\prime} / P^{\prime}\right)_{a}^{\times}$such that for any $y_{0} \in \Omega\left(x_{0}\right)$ we have $p_{\lambda}\left(x_{0}^{-1} y_{0} x\right) \notin I\left(\mathscr{T}^{\prime}\right) \cap S_{\lambda}^{2}\left(V_{1}\right)^{*}$ for all weights $\lambda$ of $V_{2}$, and $q\left(x_{0}^{-1} y_{0} x\right) \notin I_{0}$ if $r=7$.

Proof. We begin with pointing out the following useful fact. Let $\operatorname{Ver}_{\lambda}$ be the composition of the second Veronese embedding $V_{1} \rightarrow S^{2}\left(V_{1}\right)$ with the projection of $S^{2}\left(V_{1}\right)$ to its direct summand $S_{\lambda}^{2}\left(V_{1}\right)$. By Lemma 3.3, $p_{\lambda}(x)=0$ is the only quadratic equation of $G^{\prime} / P^{\prime}$ of weight $\lambda$; thus $\operatorname{Ver}_{\lambda}\left(\left(G^{\prime} / P^{\prime}\right)_{a}\right)$ spans a codimension 1 subspace of $S_{\lambda}^{2}\left(V_{1}\right)$, namely, the zero set of the linear form $p_{\lambda}(x) \in S_{\lambda}^{2}\left(V_{1}\right)^{*}$.

Next, we claim that the quadratic forms $p_{\lambda}\left(x_{0}^{-1} y_{0} x\right), y_{0} \in\left(G^{\prime} / P^{\prime}\right)_{a}^{\times}$, span a codimension 1 subspace of $S_{\lambda}^{2}\left(V_{1}\right)^{*}$. Using (8) we write

$$
p_{\lambda}\left(x_{0}^{-1} y_{0} x\right)=\sum_{\lambda=\mu+v} p_{\mu \nu} \frac{y_{0 \mu} y_{0 v}}{x_{0 \mu} x_{0 v}} x_{\mu} x_{\nu} .
$$


Suppose that for some coefficients $c_{\mu \nu}$ we have a linear relation

$$
\sum_{\lambda=\mu+v} c_{\mu \nu} p_{\mu \nu} \frac{y_{0 \mu} y_{0 v}}{x_{0 \mu} x_{0 v}}=0
$$

This can be read as a relation with coefficients $c_{\mu \nu} p_{\mu \nu} x_{0 \mu}^{-1} x_{0 v}^{-1}$ satisfied by all the vectors $\left(y_{0 \mu} y_{0 v}\right)$, where $y_{0} \in\left(G^{\prime} / P^{\prime}\right)_{a}^{\times}$and $\mu+v=\lambda$. The set of these vectors is precisely $\operatorname{Ver}_{\lambda}\left(\left(G^{\prime} / P^{\prime}\right)_{a}^{\times}\right)$. The linear span of $\operatorname{Ver}_{\lambda}\left(\left(G^{\prime} / P^{\prime}\right)_{a}^{\times}\right)$is the same as the linear span of $\operatorname{Ver}_{\lambda}\left(\left(G^{\prime} / P^{\prime}\right)_{a}\right)$. By the argument in the beginning of the proof, up to a multiplicative constant there is only one linear relation satisfied by the elements of $\operatorname{Ver}_{\lambda}\left(\left(G^{\prime} / P^{\prime}\right)_{a}\right)$, namely the one with coefficients $p_{\mu \nu}$. Therefore, $c_{\mu \nu}=x_{0 \mu} x_{0 \nu}$ is uniquely determined up to a multiplicative constant. This proves our claim. Note that the linear span under discussion is thus the space of forms vanishing at $x_{0}$.

It follows that the set of $\bar{k}$-points $y \in\left(G^{\prime} / P^{\prime}\right)_{a}^{\times}$such that $p_{\lambda}\left(x_{0}^{-1} y x\right)$ belongs to the codimension 2 subspace $I\left(\mathscr{T}^{\prime}\right) \cap S_{\lambda}^{2}\left(V_{1}\right)^{*}$, is a proper closed subset of $\left(G^{\prime} / P^{\prime}\right)_{a}^{\times}$. For $r<7$ we define $\Omega\left(x_{0}\right)$ as the complement to the union of these closed subsets for all weights $\lambda$ of $V_{2}$.

For the rest of the proof we let $r=7$. Let

$$
\operatorname{Ver}_{0}^{3}: V_{1} \rightarrow S_{0}^{3}\left(V_{1}\right)
$$

be the composition of the natural map $V_{1} \rightarrow S^{3}\left(V_{1}\right)$ with the projection $S^{3}\left(V_{1}\right) \rightarrow$ $S_{0}^{3}\left(V_{1}\right)$. The map $\operatorname{Ver}_{0}^{3}$ sends $x=\left(x_{\mu}\right)$ to the vector $\left(x_{\mu} x_{v} x_{\xi}\right)$, for all $\mu, v, \xi$ such that $\mu+v+\xi=0$. If we write the invariant cubic form (defined up to a scalar multiple) as

$$
q(x)=\sum_{\mu+v+\xi=0} q_{\mu \nu \xi} x_{\mu} x_{v} x_{\xi},
$$

then it is well known that all the coefficients $q_{\mu \nu \xi}$ are nonzero; see, for example, [Faulkner 2001]. Recall that the singular locus of the cubic hypersurface $q(x)=0$ is $\left(G^{\prime} / P^{\prime}\right)_{a}$.

Let $L_{x_{0}} \subset S_{0}^{3}\left(V_{1}\right)^{*}$ be the subspace of forms such that all their (first order) partial derivatives vanish at $x_{0}$. We claim that $L_{x_{0}}$ coincides with the linear span of the forms $q\left(x_{0}^{-1} y_{0} u\right)$, where $y_{0}$ ranges over $\left(G^{\prime} / P^{\prime}\right)_{a}$.

Let us prove this claim. The partial derivatives of $q(x)$ vanish on $\left(G^{\prime} / P^{\prime}\right)_{a}$; hence $q\left(x_{0}^{-1} y_{0} u\right) \in L_{x_{0}}$ for any $y_{0} \in\left(G^{\prime} / P^{\prime}\right)_{a}$. Thus the linear span of the forms $q\left(x_{0}^{-1} y_{0} u\right)$, where $y_{0} \in\left(G^{\prime} / P^{\prime}\right)_{a}$, is contained in $L_{x_{0}}$. We now prove that these spaces have the same dimension.

Let $f(x)=\sum_{\mu+\nu+\xi=0} f_{\mu \nu \xi} x_{\mu} x_{\nu} x_{\xi}$ be a form in $L_{x_{0}}$. The partial derivative with respect to $x_{\xi}$ is

$$
3 \sum_{\mu+\nu=-\xi} f_{\mu \nu \xi} x_{\mu} x_{v}
$$


It vanishes at $x_{0} \in V_{1}^{\times}$if and only if

$$
x_{\xi} \sum_{\mu+\nu=-\xi} f_{\mu \nu \xi} x_{\mu} x_{\nu}=\sum_{\mu+\nu=-\xi} q_{\mu \nu \xi}^{-1} f_{\mu \nu \xi} \cdot q_{\mu \nu \xi} x_{\mu} x_{\nu} x_{\xi}
$$

does. Hence $\left(q_{\mu \nu \xi}^{-1} L_{x_{0}}\right)^{\perp}$ is spanned by the 27 vectors $\left(q_{\mu \nu \xi} x_{0 \mu} x_{0 \nu} x_{0 \xi}\right)$, where $\xi$ is fixed, and $\mu, v$ are arbitrary. Since the coordinates of $x_{0}$ are not zero, this space has the same dimension as the space $M \subset S_{0}^{3}\left(V_{1}\right)$ spanned by the 27 vectors $\left(q_{\mu \nu \xi}\right)$, where $\xi$ is fixed, and $\mu, v$ are arbitrary weights satisfying $\mu+v+\xi=0$. The fact that the ideal of $\left(G^{\prime} / P^{\prime}\right)_{a}$ is generated by the partial derivatives of $q(x)$, implies that $M^{\perp}$ is the linear span of $\operatorname{Ver}_{0}^{3}\left(\left(G^{\prime} / P^{\prime}\right)_{a}\right)$. We conclude that $\operatorname{dim} L_{x_{0}}$ equals the dimension of this linear span. Since all the coefficients $q_{\mu \nu \xi}$ are nonzero, the forms $q\left(x_{0}^{-1} y_{0} u\right)$, where $y_{0} \in\left(G^{\prime} / P^{\prime}\right)_{a}$, span the space of the same dimension. This proves our claim.

We complete the proof of the proposition in the case $r=7$. A cubic form $f \in S_{0}^{3}\left(V_{1}\right)^{*}$ is in $L_{x_{0}}$ if and only if $f(x)=0$ is singular at $x_{0} \in V_{1}^{\times}$. This is the case if and only if the corresponding hyperplane $H_{f} \subset S_{0}^{3}\left(V_{1}\right)$ contains the tangent space $\Phi$ to $\operatorname{Ver}_{0}^{3}\left(V_{1}\right)$ at the point $m=\operatorname{Ver}_{0}^{3}\left(x_{0}\right)$. We have a commutative diagram (compare (15) and (2))

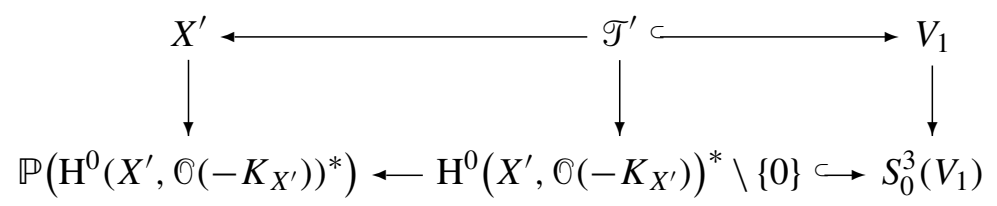

where the left-hand vertical map is the anticanonical embedding of $X^{\prime}$, and the other two are $\operatorname{Ver}_{0}^{3}$. The image of $\mathscr{T}^{\prime}$ in the 4-dimensional vector space

$$
\mathrm{H}^{0}\left(X^{\prime}, \mathcal{O}\left(-K_{X^{\prime}}\right)\right)^{*}=\left(k\left[\mathscr{T}^{\prime}\right] \cap S_{0}^{3}\left(V_{1}\right)^{*}\right)^{*}=\left(S_{0}^{3}\left(V_{1}\right)^{*} / I_{0}\right)^{*} \simeq \mathbb{A}^{4} \subset S_{0}^{3}\left(V_{1}\right)
$$

is the affine cone $X_{a}^{\prime}$ (without 0 ) over the cubic surface $X^{\prime} \subset \mathbb{P}^{3}$.

By the induction assumption $I\left(\mathcal{T}^{\prime \times}\right)$ is generated by its graded components $I_{\lambda}$ of degree 2 and weight $\lambda$, for all weights $\lambda$ of $V_{2}$. The weights of $V_{1}$ are the negatives of the weights of $V_{2}$, so $x_{-\lambda} I_{\lambda}$ has degree 3 and weight 0 . Since the coordinates $x_{-\lambda}$ are invertible on $\mathscr{T}^{\prime \times}$, the ideal $I\left(\mathcal{T}^{\prime \times}\right)$ is generated by its graded component of degree 3 and weight 0 . Hence locally in the neighborhood $\mathscr{T}^{\prime \times}$ of $x_{0}$ the ideal $I\left(\mathscr{T}^{\prime}\right)$ is generated by $I_{0}$, that is, by the equations of $\mathbb{A}^{4}$ in $S_{0}^{3}\left(V_{1}\right)$.

This implies that the tangent space $T_{X_{a}^{\prime}, m} \subset \mathbb{A}^{4}$ is $\Phi \cap \mathbb{A}^{4}$. Thus for any $f$ in a dense open subset of $L_{x_{0}}$ we have $H_{f} \cap \mathbb{A}^{4}=T_{X_{a}^{\prime}, m}$. Since $X^{\prime} \subset \mathbb{P}^{3}$ is a smooth cubic surface, $X_{a}^{\prime} \backslash T_{X_{a}^{\prime}, m}$ is dense and open in $X_{a}^{\prime}$. Therefore, for the general $f \in L_{x_{0}}$ we have $X_{a}^{\prime} \cap H_{f} \neq X_{a}^{\prime}$, so $f \notin I_{0}$. Now the above claim implies the statement of the proposition. 
Corollary 6.3. For any k-point $y_{0} \in \Omega\left(x_{0}\right)$ and any weight $\lambda$ of $V_{2}$ the closed subset of $\mathcal{T}^{\prime}$ given by $p_{\lambda}\left(x_{0}^{-1} y_{0} x\right)=0$ is the preimage $f^{\prime-1}\left(C_{\lambda}\right)$ of a geometrically integral $k$-conic $C_{\lambda} \subset X^{\prime}$ passing through $M_{r}$. For $r=7$ the closed subset of $\mathscr{T}^{\prime}$ given by $q\left(x_{0}^{-1} y_{0} x\right)=0$, for any $y_{0} \in \Omega\left(x_{0}\right)$, is the preimage $f^{\prime-1}(Q)$ of a geometrically integral cubic $k$-curve $Q$ with a double point at $M_{r}$ (the intersection of the cubic surface $X^{\prime}$ with its tangent plane at $M_{r}$ ).

Proof. To check that $M_{r} \in C_{\lambda}$, set $x=x_{0}$; then $p_{\lambda}\left(x_{0}^{-1} y_{0} x\right)=p_{\lambda}\left(y_{0}\right)=0$ by Lemma 3.3 since $y_{0} \in\left(G^{\prime} / P^{\prime}\right)_{a}$. If the conic $C_{\lambda}$ is not geometrically integral, then its components must have intersection index 1 with $-K_{X^{\prime}}$, so there are two of them. It is well known that a curve on $X^{\prime}$ has such a property if and only if it is an exceptional curve. However, $M_{r}$ does not belong to the exceptional curves of $X^{\prime}$. Thus $C_{\lambda}$ is geometrically integral.

If $r=7$, by substituting $x=x_{0}$ one shows as before that $Q$ contains $M_{7}$ (the cubic form $q$ vanishes on $G^{\prime} / P^{\prime}$ ). Since the $p_{\lambda}(x)$ are partial derivatives of $q(x)$, and $M_{7} \in C_{\lambda}$, we see that $Q$ has a double point at $M_{7}$. If $Q$ is not geometrically integral, then it is the union of a geometrically integral conic and an exceptional curve, or the union of three exceptional curves. In each of these cases the singular point $M_{7} \subset Q$ will have to lie on an exceptional curve, and this is a contradiction.

Corollary 6.4. For any $y_{0} \in \Omega\left(x_{0}\right)$ the scheme-theoretic intersection of $x_{0}^{-1} y_{0} \mathscr{T}^{\prime}$ and $\left(G^{\prime} / P^{\prime}\right)_{a}$ is the orbit $T^{\prime} y_{0}$.

Proof. By Lemma 3.3 the ideal of $\left(G^{\prime} / P^{\prime}\right)_{a}$ is generated by $p_{\lambda}(x)$, for all weights $\lambda$ of $V_{2}$. As was remarked at the end of Section 5, there exist weights $\lambda$ and $v$ such that the intersection index of $C_{\lambda}$ and $C_{\nu}$ on $X^{\prime}$ is 1 , that is, $M_{r}$ is the schemetheoretic intersection $C_{\lambda} \cap C_{v}$. Thus the orbit $T^{\prime} y_{0}$ is the closed subscheme of $x_{0}^{-1} y_{0} \mathscr{T}^{\prime}$ given by $p_{\lambda}(x)=p_{v}(x)=0$, and our statement follows.

Let $\sigma: X=\mathrm{Bl}_{M_{r}}\left(X^{\prime}\right) \rightarrow X^{\prime}$ be the morphism inverse to the blowing-up of $M_{r}$. Then $\sigma$ induces an isomorphism of $X \backslash \sigma^{-1}\left(M_{r}\right)$ with $X^{\prime} \backslash M_{r}$, and $\sigma^{-1}\left(M_{r}\right) \cong \mathbb{P}^{1}$. The proper transform of a curve $D \subset X^{\prime}$ is defined as the closure of $\sigma^{-1}\left(D \backslash M_{r}\right)$ in $X$. The comparison of intersection indices on $X^{\prime}$ and $X$ shows that the proper transforms of the conics $C_{\lambda}$ and the singular cubic $Q$ (for $r=7$ ) are exceptional curves on $X$. By comparing the numbers we see that these curves together with $\sigma^{-1}\left(M_{r}\right)$ and the inverse images of the exceptional curves on $X^{\prime}$ give the full set of exceptional curves on $X$.

End of proof of Theorem 6.1 Consider the open set $U \subset(G / P)_{a}$ and the morphism $\pi: U \rightarrow V_{1} \backslash\{0\}$; see Corollary 4.2. Choose any $y_{0} \in \Omega\left(x_{0}\right)$, and define $\mathscr{T} \subset U$ as the "proper transform" of $x_{0}^{-1} y_{0} \mathscr{T}^{\prime}$ with respect to $\pi$. Explicitly, $\mathscr{T} \subset U$ 
is defined as the Zariski closure of

$$
\pi^{-1}\left(x_{0}^{-1} y_{0} \mathscr{T}^{\prime} \backslash\left(G^{\prime} / P^{\prime}\right)_{a}\right)=\pi^{-1}\left(x_{0}^{-1} y_{0} \mathscr{T}^{\prime} \backslash T^{\prime} y_{0}\right),
$$

where the equality is due to Corollary 6.4. The torus $T^{\prime}$ acts on $\mathscr{T}^{\prime}$, and $\pi$ is $T^{\prime}$ equivariant; hence $T^{\prime}$ acts on $\mathscr{T}$. But $\mathbf{G}_{m}=\left\{g_{t}\right\}$ (see Lemma 4.1) also acts on $\mathscr{T}$. The torus $T$ is generated by $T^{\prime}$ and $\mathbf{G}_{m}=\left\{g_{t}\right\}$, so $T$ acts on $\mathscr{T}$.

Corollaries 4.2 and 6.4 imply that the restriction of $\pi$ to $\mathscr{T}$ is the composition of a torsor under $\mathbf{G}_{m}=\left\{g_{t}\right\}$ and the morphism $\mathrm{Bl}_{y_{0} T^{\prime}}\left(x_{0}^{-1} y_{0} \mathscr{T}^{\prime}\right) \rightarrow x_{0}^{-1} y_{0} \mathscr{T}^{\prime}$ inverse to the blowing-up of the orbit $T^{\prime} y_{0}$ in $x_{0}^{-1} y_{0} \mathscr{T}^{\prime}$. The blowing-up of $T^{\prime} y_{0}$ in $x_{0}^{-1} y_{0} \mathscr{T}^{\prime}$ is naturally isomorphic to the pullback $\mathscr{T}^{\prime} \times{ }_{X^{\prime}} X$ of the torsor $\mathscr{T}^{\prime} \rightarrow X^{\prime}$ to $X$. This can be summarized in the commutative diagram

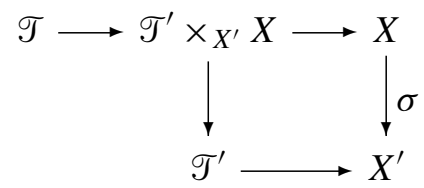

where the horizontal arrows are torsors under tori, and the vertical arrows are contractions. The composed morphism $f: \mathscr{T} \rightarrow X$ is a composition of two torsors under tori, and hence is an affine morphism whose fibres are orbits of $T$. Therefore $\mathscr{T}$ is an $X$-torsor under $T$, by Lemma 1.1. We obtain a $T$-equivariant embedding $\mathscr{T} \hookrightarrow(G / P)_{a}$.

For $r<7$ we note that $I\left(\mathcal{T}^{\times}\right) \subset k\left[V^{\times}\right]$is generated by $I\left(x_{0}^{-1} y_{0} \mathscr{T}^{\prime \times}\right)$ and the equations of $(G / P)_{a}$; moreover, for each weight $w \omega_{1}, w \in \mathrm{W}$, there is exactly one quadratic equation, by Lemma 3.3. The restriction of $\omega_{1} \in \hat{H}=P(\mathrm{R})$ to $H^{\prime}$ is again the weight $\omega_{1} \in \hat{H}^{\prime}=P\left(\mathrm{R}^{\prime}\right)$. By the induction assumption $I\left(\mathscr{T}^{\prime \times}\right)$ is generated by its graded components of degree 2 of such weights; hence the same is true for $I\left(\mathscr{T}^{\times}\right)$.

It remains to prove that $\mathscr{T} \subset(G / P)_{a}^{s f}$, and that the torsor $f: \mathscr{T} \rightarrow X$ is universal. The action of $T$ on $\mathscr{T}$ is free; we show that every point of $\mathscr{T}$ is stable. We claim that $f$ sends the weight hyperplane sections of $\mathscr{T}$ to the exceptional curves on $X$. By the results of Section 4 this follows from the induction assumption for the weights of $V_{1}$, and from Corollary 6.3 for the weights of $V_{2} \oplus V_{3}$. Corollary 6.4 implies that the highest weight hyperplane $x_{\omega}=0$ corresponds to $\sigma^{-1}\left(M_{r}\right)$. By Lemma 5.1 (ii) the set of exceptional curves of $X$ is identified with the set $\mathrm{W} \omega$ in such a way that two distinct exceptional curves intersect in $X$ if and only if the corresponding weights are not adjacent vertices of the convex hull $\operatorname{Conv}(\mathrm{W} \omega)$. Now Proposition 2.4 implies that $\mathcal{T} \subset(G / P)_{a}^{s f}$. We thus obtain an embedding $X \hookrightarrow Y$.

The pull-back of the torsor $(G / P)_{a}^{s f} \rightarrow Y$ to $X$ gives rise to the following commutative diagram, where the horizontal arrows represent the types of corresponding 
torsors

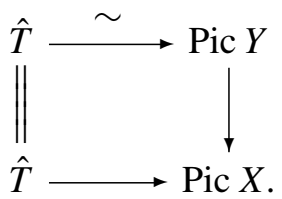

The upper horizontal arrow is an isomorphism since the torsor $(G / P)_{a}^{s f} \rightarrow Y$ is universal, by Theorem 2.7. Since the exceptional curves on $X$ are cut by divisors on $Y$, the restriction map Pic $Y \rightarrow \operatorname{Pic} X$ is surjective. However, the ranks of Pic $Y$ and Pic $X$ are equal, so this map is an isomorphism. Now it follows from the diagram that the type of the torsor $f: \mathscr{T} \rightarrow X$ is an isomorphism, so this torsor is universal as well. The theorem is proved.

\section{Acknowledgement}

The second author is grateful to Centre de recherches mathématiques de l'Université de Montréal, the Mathematical Sciences Research Institute in Berkeley, and the organizers of the special semester "Rational and integral points on higherdimensional varieties" for the hospitality and support.

\section{References}

[Batyrev and Popov 2004] V. V. Batyrev and O. N. Popov, "The Cox ring of a del Pezzo surface", pp. 85-103 in Arithmetic of higher-dimensional algebraic varieties (Palo Alto, CA, 2002), edited by B. Poonen and Y. Tschinkel, Progr. Math. 226, Birkhäuser, Boston, 2004. MR 2005h:14091 Zbl 1075.14035

[Bourbaki 1981] N. Bourbaki, Groupes et algèbres de Lie, Chapitres IV-VIII, Masson, Paris, 1981. MR 83g:17001 Zbl 0498.12001

[Colliot-Thélène and Sansuc 1987] J.-L. Colliot-Thélène and J.-J. Sansuc, "La descente sur les variétés rationnelles, II”, Duke Math. J. 54:2 (1987), 375-492. MR 89f:11082 Zbl 0659.14028

[Dabrowski 1996] R. Dabrowski, "On normality of the closure of a generic torus orbit in $G / P$ ", Pacific J. Math. 172:2 (1996), 321-330. MR 97d:14067 Zbl 0969.14034

[Derenthal 2006] U. Derenthal, "On the Cox ring of Del Pezzo surfaces", 2006. math.AG/0603111

[Derenthal 2007] U. Derenthal, "Universal torsors of del Pezzo surfaces and homogeneous spaces", Adv. Math. 213:2 (2007), 849-864. MR 2332612 Zbl pre05168539 math.AG/0604195

[Dolgachev 2003] I. Dolgachev, Lectures on invariant theory, London Mathematical Society Lecture Notes Series 296, Cambridge Univ. Press, Cambridge, 2003. MR 2004g:14051 Zbl 1023.13006

[Faulkner 2001] J. R. Faulkner, "Generalized quadrangles and cubic forms", Comm. Algebra 29:10 (2001), 4641-4653. MR 2002j:17039 Zbl 0992.17017

[Flaschka and Haine 1991] H. Flaschka and L. Haine, "Torus orbits in G/P", Pacific J. Math. 149:2 (1991), 251-292. MR 92e:14047 Zbl 0788.22017

[Friedman and Morgan 2002] R. Friedman and J. W. Morgan, "Exceptional groups and del Pezzo surfaces", pp. 101-116 in Symposium in Honor of C. H. Clemens, edited by A. Bertram et al., Contemp. Math. 312, Amer. Math. Soc., Providence, RI, 2002. MR 2004b:14061 Zbl 1080.14533 
[Gel'fand and Serganova 1987] I. M. Gel'fand and V. V. Serganova, "Combinatorial geometries and the strata of a torus on homogeneous compact manifolds", Uspekhi Mat. Nauk 42:2(254) (1987), 107-134, 287. MR 89g:32049 Zbl 0629.14035

[Hartshorne 1977] R. Hartshorne, Algebraic geometry, Graduate Texts in Mathematics 52, Springer, New York, 1977. MR 57 \#3116 Zbl 0367.14001

[Helgason 2001] S. Helgason, Differential geometry, Lie groups, and symmetric spaces, Graduate Studies in Mathematics 34, American Mathematical Society, Providence, RI, 2001. Corrected reprint of the 1978 original. MR 2002b:53081 Zbl 0993.53002

[Lancaster and Towber 1979] G. Lancaster and J. Towber, "Representation-functors and flag-algebras for the classical groups. I”, J. Algebra 59:1 (1979), 16-38. MR 80i:14020 Zbl 0441.14013

[Leung 2000] N. C. Leung, "ADE-bundles over rational surfaces, configuration of lines and rulings", preprint, 2000. math.AG/0009192

[Manin 1986] Y. I. Manin, Cubic forms: algebra, geometry, arithmetic, 2nd ed., North-Holland Mathematical Library 4, North-Holland, Amsterdam, 1986. MR 87d:11037 Zbl 0582.14010

[Manivel 2006] L. Manivel, "Configurations of lines and models of Lie algebras", J. Algebra 304:1 (2006), 457-486. MR 2007f:17017 Zbl pre05078835

[Mumford et al. 1994] D. Mumford, J. Fogarty, and F. Kirwan, Geometric invariant theory, 3rd ed., Ergebnisse der Mathematik 34, Springer, Berlin, 1994. MR 95m:14012 Zbl 0797.14004

[Onishchik and Vinberg 1990] A. L. Onishchik and È. B. Vinberg, Lie groups and algebraic groups, Springer, Berlin, 1990. MR 91g:22001 Zbl 0722.22004

[Popov 1974] V. L. Popov, "Picard groups of homogeneous spaces of linear algebraic groups and one-dimensional homogeneous vector fiberings", Izv. Akad. Nauk SSSR Ser. Mat. 38 (1974), 294322. MR 50 \#9867 Zbl 0298.14023

[Popov 2001] O. N. Popov, Diplomarbeit, Universität Tübingen, 2001.

[Skorobogatov 1993] A. N. Skorobogatov, "On a theorem of Enriques-Swinnerton-Dyer", Ann. Fac. Sci. Toulouse Math. (6) 2:3 (1993), 429-440. MR 95b:14018 Zbl 0811.14020

[Skorobogatov 2001] A. Skorobogatov, Torsors and rational points, Cambridge Tracts in Mathematics 144, Cambridge University Press, Cambridge, 2001. MR 2002d:14032 Zbl 0972.14015

Communicated by Bjorn Poonen

Received 2007-02-02 Revised 2007-08-11 Accepted 2007-09-15

serganov@math.berkeley.edu Department of Mathematics, University of California, Berkeley, CA 94720-3840, United States

a.skorobogatov@imperial.ac.uk Department of Mathematics, South Kensington Campus, Imperial College, London SW72BZ, United Kingdom

Institute for Information Transmission Problems, Russian Academy of Sciences, 19 Bolshoi Karetnyi, Moscow, 127994, Russia 


\title{
Algebra \& Number Theory
}

www.jant.org

\section{EDITORS}

\section{MANAGING EDITOR}

Bjorn Poonen

University of California

Berkeley, USA

\author{
EDITORIAL BOARD CHAIR \\ David Eisenbud \\ University of California \\ Berkeley, USA
}

\section{BOARD OF EDITORS}

\section{Georgia Benkart}

Dave Benson

Richard E. Borcherds

John H. Coates

J-L. Colliot-Thélène

Brian D. Conrad

Hélène Esnault

Hubert Flenner

Edward Frenkel

Andrew Granville

Joseph Gubeladze

Ehud Hrushovski

Craig Huneke

Mikhail Kapranov

Yujiro Kawamata

János Kollár

Hendrik W. Lenstra

Yuri Manin

Barry Mazur
University of Wisconsin, Madison, USA

University of Aberdeen, Scotland

University of California, Berkeley, USA

University of Cambridge, UK

CNRS, Université Paris-Sud, France

University of Michigan, USA

Universität Duisburg-Essen, Germany

Ruhr-Universität, Germany

University of California, Berkeley, USA

Université de Montréal, Canada

San Francisco State University, USA

Hebrew University, Israel

University of Kansas, USA

Yale University, USA

University of Tokyo, Japan

Princeton University, USA

Universiteit Leiden, The Netherlands

Northwestern University, USA

Harvard University, USA
Susan Montgomery

Shigefumi Mori

Andrei Okounkov

Raman Parimala

Victor Reiner

Karl Rubin

Peter Sarnak

Michael Singer

Ronald Solomon

Vasudevan Srinivas

J. Toby Stafford

Bernd Sturmfels

Richard Taylor

Ravi Vakil

Michel van den Bergh

Marie-France Vignéras

Kei-Ichi Watanabe

Andrei Zelevinsky

Efim Zelmanov
University of Southern California, USA

RIMS, Kyoto University, Japan

Princeton University, USA

Emory University, USA

University of Minnesota, USA

University of California, Irvine, USA

Princeton University, USA

North Carolina State University, USA

Ohio State University, USA

Tata Inst. of Fund. Research, India

University of Michigan, USA

University of California, Berkeley, USA

Harvard University, USA

Stanford University, USA

Hasselt University, Belgium

Université Paris VII, France

Nihon University, Japan

Northeastern University, USA

University of California, San Diego, USA

\section{PRODUCTION}

ant@mathscipub.org

Paulo Ney de Souza, Production Manager

Silvio Levy, Senior Production Editor

See inside back cover or www.jant.org for submission instructions.

The subscription price for 2007 is US \$120/year for the electronic version, and \$180/year for print and electronic. Subscriptions, requests for back issues from the last three years and changes of subscribers address should be sent to Mathematical Sciences Publishers, Department of Mathematics, University of California, Berkeley, CA 94720-3840, USA.

Algebra \& Number Theory (ISSN 1937-0652) at Mathematical Sciences Publishers, Department of Mathematics, University of California, Berkeley, CA 94720-3840 is published continuously online. Periodical rate postage paid at Berkeley, CA 94704, and additional mailing offices.

ANT peer review and production are managed by EditFLOW ${ }^{\mathrm{TM}}$ from Mathematical Sciences Publishers.

\author{
PUBLISHED BY \\ mathematical sciences publishers \\ http://www.mathscipub.org \\ A NON-PROFIT CORPORATION \\ Typeset in LATEX
}

Copyright $\odot 2007$ by Mathematical Sciences Publishers 


\section{Algebra \& Number Theory}

Volume $1 \quad$ No. $4 \quad 2007$

Finite descent obstructions and rational points on curves MiCHAEL STOLL

Del Pezzo surfaces and representation theory

Vera V. Serganova and AleXei N. Skorobogatov

The zeta function of monomial deformations of Fermat hypersurfaces

REMKE KLOOSTERMAN

Dual graded graphs for Kac-Moody algebras 